\title{
The zebrafish progranulin gene family and antisense transcripts Benoît Cadieux ${ }^{1,2}$, Babykumari P Chitramuthu ${ }^{1}$, David Baranowski ${ }^{1}$ and Hugh PJ Bennett*1,3
}

Address: ${ }^{1}$ Endocrine Laboratory, Royal Victoria Hospital, McGill University Health Centre, Montreal, Quebec, Canada, ${ }^{2}$ Cancer Research Institute, UCSF, 2340 Sutter Street, N-231 San Francisco, CA 94143, USA and ${ }^{3}$ Room L2.05, Royal Victoria Hospital, 687 Pine Avenue West, Montreal, Quebec, H3A 1A1, Canada

Email: Benoît Cadieux - bcadieux@cc.ucsf.edu; Babykumari P Chitramuthu - babykumari.perumal1@mcgill.ca; David Baranowski - dbaran3@po-box.mcgill.ca; Hugh PJ Bennett* - hugh.bennett@mcgill.ca

* Corresponding author

Published: 08 November 2005

BMC Genomics 2005, 6:156 doi:10.1186/147|-2164-6-156
Received: 29 August 2005

Accepted: 08 November 2005

This article is available from: http://www.biomedcentral.com/I47I-2/64/6/156

(c) 2005 Cadieux et al; licensee BioMed Central Ltd.

This is an Open Access article distributed under the terms of the Creative Commons Attribution License (http://creativecommons.org/licenses/by/2.0), which permits unrestricted use, distribution, and reproduction in any medium, provided the original work is properly cited.

\begin{abstract}
Background: Progranulin is an epithelial tissue growth factor (also known as proepithelin, acrogranin and PC-cell-derived growth factor) that has been implicated in development, wound healing and in the progression of many cancers. The single mammalian progranulin gene encodes a glycoprotein precursor consisting of seven and one half tandemly repeated non-identical copies of the cystine-rich granulin motif. A genome-wide duplication event hypothesized to have occurred at the base of the teleost radiation predicts that mammalian progranulin may be represented by two co-orthologues in zebrafish.
\end{abstract}

Results: The cDNAs encoding two zebrafish granulin precursors, progranulins-A and $-B$, were characterized and found to contain 10 and 9 copies of the granulin motif respectively. The cDNAs and genes encoding the two forms of granulin, progranulins-I and -2 , were also cloned and sequenced. Both latter peptides were found to be encoded by precursors with a simplified architecture consisting of one and one half copies of the granulin motif. A cDNA encoding a chimeric progranulin which likely arises through the mechanism of trans-splicing between grn I and grn2 was also characterized. A non-coding RNA gene with antisense complementarity to both grn I and grn2 was identified which may have functional implications with respect to gene dosage, as well as in restricting the formation of the chimeric form of progranulin. Chromosomal localization of the four progranulin (grn) genes reveals syntenic conservation for grna only, suggesting that it is the true orthologue of mammalian grn. RT-PCR and whole-mount in situ hybridization analysis of zebrafish grns during development reveals that combined expression of grna and grnb, but not grn I and grn2, recapitulate many of the expression patterns observed for the murine counterpart. This includes maternal deposition, widespread central nervous system distribution and specific localization within the epithelial compartments of various organs.

Conclusion: In support of the duplication-degeneration-complementation model of duplicate gene retention, partitioning of expression between grna and grnb was observed in the intermediate cell mass and yolk syncytial layer, respectively. Taken together these expression patterns suggest that the function of an ancestral grn gene has been devolved upon four paralogues in zebrafish. 


\section{Background}

In mammals, a single progranulin gene, also known as proepithelin, acrogranin and PC-cell-derived growth factor (PCDGF), encodes a glycoprotein precursor exhibiting pleiotropic tissue growth factor activity (reviewed in [1$4]$ ). Progranulin is secreted in an intact form [5-8], or undergoes proteolysis leading to the release of its constituent peptides, the granulins [9-11]. Individual granulins have an approximate molecular weight of $6 \mathrm{kDa}$, and are structurally defined by the presence of 12 cysteines arranged in a characteristic motif: $\mathrm{X}_{2-3} \underline{\mathrm{CX}_{5-}}$ ${ }_{6} \mathrm{CX}_{5} \underline{\mathrm{CCX}}_{8} \mathrm{CCX}_{6} \underline{\mathrm{CCX}}{ }_{5} \mathrm{CCX}_{4} \mathrm{CX}_{5-6} \underline{\mathrm{CX}}_{2}$ [12]. Comparison of the biosynthetic origin of granulin peptides in various mammals reveals that all are commonly derived from a precursor composed of one amino-terminal half followed by seven non-identical copies of the granulin motif.

A role of progranulin in mammalian embryogenesis has been suggested. The exogenous addition of recombinant progranulin to eight-cell stage mouse embryos grown in culture ex vivo accelerates the onset of cavitation, stimulates the rate of blastocoel expansion, and leads to an increase in the number of trophectoderm cells compared to controls [13]. Conversely, the use of a progranulin function-blocking antibody arrests growth and prohibits embryo implantation $[13,14]$. These results are consistent with the growth-promoting activity of progranulin upon epithelial cells in vitro.

Despite these advances, the evolutionary history and phylogenetic distribution of the progranulin gene outside the mammalian radiation remain largely unexplored. In order to shed light on this issue, and to establish a model for studying the functional contribution of progranulin to vertebrate development, we undertook the characterization of the biosynthetic origins of progranulins in the zebrafish. The widely documented evidence in favor of a pan-genomic duplication event at the base of the teleost radiation [15], commonly referred to as $3 \mathrm{R}$, predicts that the single mammalian progranulin gene will likely be represented by two zebrafish co-orthologues $[16,17]$. Results reported here demonstrate that, in zebrafish, progranulins arise as members of an extended gene family represented by two distinct architectures, in excess of that predicted by 3R.

Comparative chromosomal mapping of the various progranulin genes (grns) was performed to assist in the establishment of an orthologous relationship to their mammalian counterpart and to provide a point of reference to discuss the evolutionary origins of the distinct progranulin architectures. In support of the duplicationdegeneration-complementation (DDC) model [18] gene expression analysis of the zebrafish progranulins reveals spatio-temporal divergence among the different family members, possibly reflecting extensive functional devolution of an ancestral form. Also, the occurrence of natural antisense transcription to some grns suggests that gene dosage may have influenced the retention of extra grn paralogues in zebrafish.

\section{Results}

Evidence for a progranulin multigene family in teleosts

A previous study indicated that major forms of granulin peptides found in hematopoietic organs of carp, Cyprinus carpio, differ in their relative abundance [19]. Specifically, extracts of carp spleen contained granulin-1 only, while the head kidney contained granulins- $1,-2$, and -3 , arguing that some granulins found in teleost fish, unlike those found in mammals, have different biosynthetic origins [19]. We revisited the issue of the sole occurrence of granulin-1 in carp spleen and confirmed the earlier report. However, granulin-1 was found to co-purify with another member of the granulin family (Figure 1, Panel A). Sequencing of this peptide suggested that it is a close homologue of mammalian granulin-A, sharing 58\% iden-

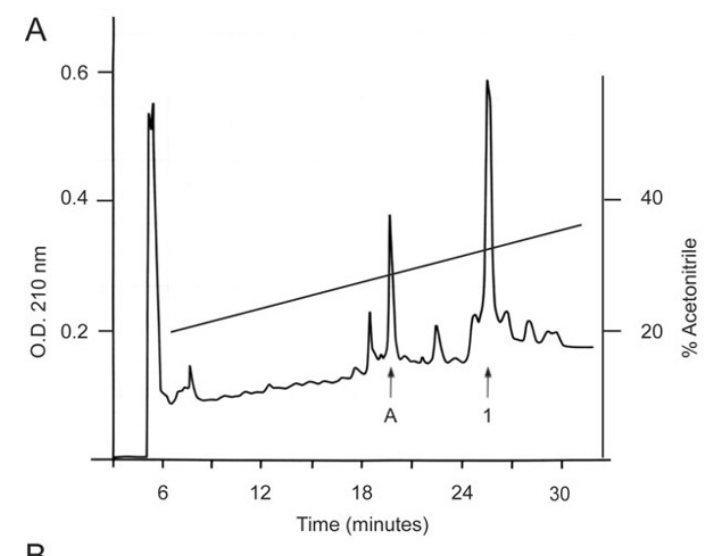

B

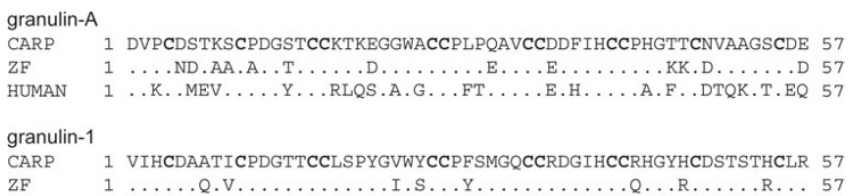

\section{Figure I}

Reversed-Phase HPLC purification of granulin-A and granulin-I from extracts of two carp spleens. Panel A: HPLC fractions derived from an extract of two carp spleens and enriched in granulin-I immunoreactivity were further purified by HPLC using $0.13 \%$ heptafluorobutyric acid as counterion. Two major components were identified, a peptide sharing $58 \%$ sequence conservation with human granulin-A (peak A) and granulin-I (peak I), each indicated by arrows. Panel B: Sequence comparison of carp granulin peptides with their respective candidate orthologues deduced from cloned zebrafish cDNA sequences (this study), and human granulin-A. Numbers correspond to amino acid position. Characteristic cysteines are shown in bold. 


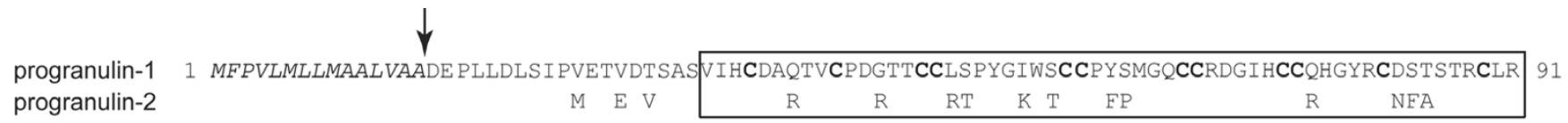

progranulin-1 92 GWLTLPSSFQKATRTFQKDQTHAETVQCEGNFYCPAEKFCCKTRTGQWGCCSGLEL. 147

$\begin{array}{llllllllll}\text { progranulin-2 } & S & E & E & Q & G & E\end{array}$

Figure 2

Comparison of the deduced translated sequences for zebrafish progranulin-I and progranulin-2. Two zebrafish cDNAs sharing $92.3 \%$ identity (grn I and grn2), each possessing a $44 \mathrm{I}$ nucleotide-long open reading frame (ORF), encode deduced precursors consisting of one full and one amino-terminal half granulin peptide, with a calculated mass of I4.5 kDa and I4.8 kDa, respectively. Both carry an identical signal peptide (italics), whose predicted cleavage site is indicated by an arrow. The predicted sequences for zebrafish granulins I and 2 are highlighted. Numbers correspond to amino acid position.

tity with the human peptide (Figure 1, Panel B). The isolation of a carp granulin peptide homologous to mammalian granulin-A suggested that teleosts synthesize a protein equivalent to mammalian progranulin. Carp is a tetraploid species known to express multiple copies of closely related genes that can complicate the study of the origins of multigene families [20]. Therefore, we chose to study the structure and expression of granulin genes in the zebrafish, a closely-related diploid teleost of the cyprinoforme order [21].

\section{Zebrafish progranulins are represented by two distinct architectures \\ Progranulin-I and progranulin-2}

A PCR-based strategy using degenerate primers based on the amino acid sequence of carp granulin-1 led to the cloning of two zebrafish cDNAs sharing 92.3\% identity and encoding structures homologous to carp granulin-1 and granulin-2, respectively (see Materials and Methods, Figure 2 and Additional File 1). In contrast with mammalian progranulins, the deduced architecture of these zebrafish precursors, named progranulin-1 (grn1) and progranulin-2 (grn2), consist of one full and one aminoterminal half granulin-like repeats only (Figure 2). Unlike progranulins A and B (see below), no potential N-glycosylation sites are found within the predicted progranulin1 or progranulin-2 polypeptide structures. Subsequent cloning of the respective genes for grn 1 and grn 2 revealed that each is encoded on five exons (as shown for progranulin-1 in Additional Files 2 and 3), confirming that they are derived from distinct transcriptional units. The genomic sequences encoding grn 1 and grn2 are interrupted by short introns in the exact equivalent positions to that observed within the mammalian grn gene [22,23]. Sequences at the splice junctions for the grn1, grn 2 and ASgrn1-2 genes are depicted in Additional File 4.

\section{Hybrid grn RNA}

A cDNA encoding a hybrid progranulin identical in size to, and sharing 95.6\% nucleotide sequence identity with both grn 1 and grn2, was uncovered through our cloning strategy (see Materials and Methods). The nucleotide substitutions for hybrid progranulin are non-randomly distributed among the exons for grn 1 and grn2, indicating that it is likely derived from the joining of the first two exons of grn 1 with exons 3, 4 and 5 of grn2 (Figure 3 and Additional File 5). A hybrid of the opposite character was not detected (i.e. exons 1 and 2 of grn 2 fused to exons 3, 4 and 5 of grn 1 ). No evidence was found to suggest the existence of additional genomic sequences corresponding to grn 1 or grn2, raising the possibility of a post-transcriptional mechanism underlying the origin of this chimeric structure.

\section{Antisense progranulin I-2 gene}

During the cloning of grn 1 , genomic sequences were used to perform BLAST searches for sequences deposited at NCBI [24]. An EST was detected (GenBank accession AW777232) whose sequence was an exact match to a portion of the grn 1 gene, but in the reverse complement orientation. The full characterization of this EST, designated ASgrn1-2, revealed that it is spliced and shares exonic complementarity to exons 2 and 3, in addition to flanking intronic sequences, of both grn 1 and grn 2 genes (Figure 3 and Additional File 6). This observation suggested that grn2 is located upstream of grn 1 in a head-to-tail organization, thus providing strong support for trans-splicing between the grn 1 and grn 2 primary transcripts as a mechanism for the genesis of the hybrid progranulin RNA (Figure 3).

In addition to its partial complementarity to grn 1 and grn2, the last exon of the ASgrn1-2 gene (corresponding to nucleotides 1015 to 1989 of the cDNA) shares a high degree of sequence conservation with the tzf transposon [25], a subclass belonging to the Tc1/mariner superfamily of class II DNA mobile elements [25]. However, this mobile element is in the reverse complement orientation within the antisense transcript, and has undergone extensive mutations resulting from nucleotide insertions, dele- 
hybrid grn transcript
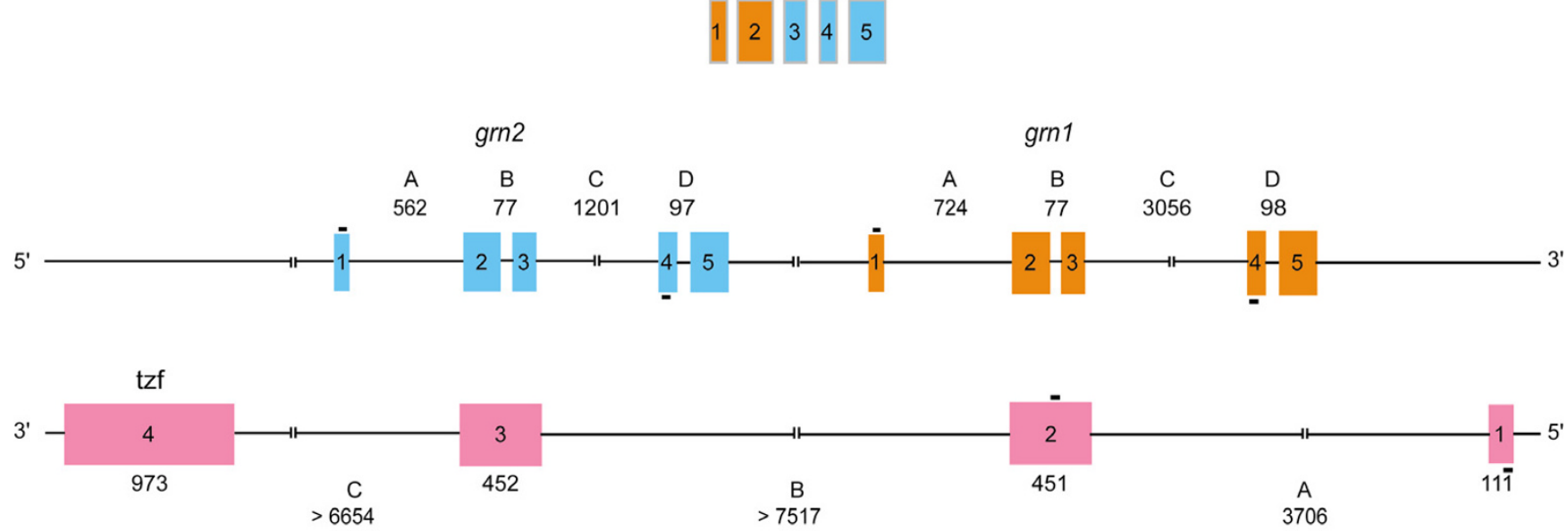

ASgrn1-2

Figure 3

Genomic organization of zebrafish grn I, grn2 and their complementary antisense gene. Zebrafish grn I and grn2 genes are found in tandem in a head-to-tail orientation and share an identical exonic organization (exons $1-5$, orange and blue boxes, respectively), but differ in their respective intron lengths (A, C, D). The spliced and polyadenylated non-protein coding ASgrn I-2 is encoded on four exons (pink boxes) and shares exon/intron complementarity to both grn I and grn2 (see text for details). Shown on top is a schematic representation of the chimeric progranulin transcript, suggesting trans-splicing as a mechanism for its generation. Bars (-) above or below exons indicate the relative position of primer pairs (grn I +2 forward and reverse; ASgrn I-2 forward and reverse) used for discriminating between ASgrn I-2 and combined grn I/grn2 expression using RT-PCR.

tions and point mutations (Additional File 7). Thus, ASgrn1-2 never possessed the ability to encode a translatable transposase protein, nor does it have a clearly predictable ORF in view of the presence of several termination codons in all three possible reading frames. For these reasons, this naturally occurring antisense transcript is considered to belong to the category of non-coding RNA genes.

The zebrafish co-orthologues of mammalian progranulin

Based on the finding of a carp granulin peptide whose sequence was homologous to mammalian forms of granulin (Figure 1, panel B), it was reasoned that these observations could be extended to the zebrafish. Using a strategy identical to that used for cloning a cDNA encoding granulin-1, we isolated a partial cDNA encoding a deduced zebrafish granulin-A peptide (data not shown). BLAST searches using this sequence retrieved two distinct ESTs from GenBank databases (accession AW174591 and AW184435), the former being identical to our cloned sequence. Sequencing of these cDNAs, referred to as progranulin-a (grna) and progranulin-b (grnb) respectively, revealed that each encodes a deduced granulins precursor bearing 10 and 9 tandemly repeated and non-identical granulin domains (Figure 4), demonstrating that the zebrafish progranulin repertoire is not limited to the simplified grn gene structures.

Zebrafish progranulin-A and progranulin-B are $48.6 \%$ identical over their aligned sequences, and each is similarly related to human progranulin with $44.8 \%$ and $42.9 \%$ identity, respectively. As expected, sequence conservation is seen primarily within the aligned granulin domains of the zebrafish precursors (Figure 4). However, the deduced granulins within the zebrafish grnA and grnB precursors cannot be aligned strictly on the basis of the mammalian nomenclature (i.e. in the order granulin- $\mathrm{G}_{\text {, - }}$ F, -B, -A, -C, -D, -E) [5,26-28]. Indeed, progranulin-A contains five granulin peptides (domains $4,6,7,8,9$ ) whose sequences bear close sequence similarity with oneanother and with that of human granulin-A. In addition, zebrafish progranulin-A encodes a deduced granulin structure (domain 2) displaying the modified cysteine motif found in human granulin $\mathrm{G}$, which is characterized by the absence of cysteine residues 4 and 7 (Figure 4).

\section{Northern blot analysis}

The size of the cloned sense and antisense zebrafish progranulin transcripts was assessed by northern blot analysis (Additional File 8). With the exception of the putative 


\begin{abstract}
Granulin
Domain

ZF progranulin-A Human progranulin

ZF progranulin-A ZF progranulin-B Human progranulin

ZF progranulin-A ZF progranulin-B Human progranulin

ZF progranulin-A ZF progranulin-B Human progranulin

ZF progranulin-A ZF progranulin- $B$ Human progranulin

ZF progranulin-A ZF progranulin-B Human progranulin

ZF progranulin-A ZF progranulin-B Human progranulin

ZF progranulin-A ZF progranulin-B Human progranulin

ZF progranulin-A ZF progranulin-B Human progranulin

ZF progranulin-A ZF progranulin-B Human progranulin

MLR-LTVCLAVVTLVICS -QCPDNEVCEAG QSCCQDPTGGFSCCPFHHGECCEDHLHCCPEGMLCS VKDLTCTNATHTEPLAD 81 MVRAAFIALLCVCVNACTA LICPDGGMCEDE NTCCLTPSGGYGCCPLPHAECCSDHLHCCYQGTLCD LEHSKCVN KTHVLDWVE 84 MWT-LVSWVALTAGLV --RTQAKKPDLPKSFRMIFSMPASESISCPDG SSCPAEFSCLLMSTSYGCCPVAQGLACSDGKHCCPN DHECSSDSSLCVRRKVKV 166 2 KVEAK-----

EFVLCGNGTS ECPADTTCCQAEDGLWG-CCPMPKAVCCDDKIHCCPEDTVCDVKALKCISSTNQELPMWDKF PARLRAEWEDHKQKKPET 255 3 A AVVCPDGESECPDDTTCCQMPDGGWG-CC PMKNAVCCDDRKHCCPQGTTCDLVHSMCVSATYGSS PFLRKFAARRRKPLEKN------- 172

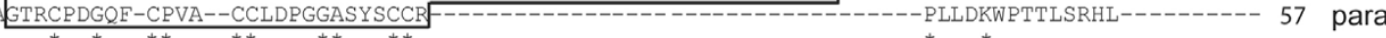

QRTTTRPTGTTSTNTAANQMT TLPAEHQAVS $\$$ DVPCND-TAACADGTTCCKTKDGGWACCPLPEAVCCEDFIHCCPHGKKCDVAAGSCDD 344 4 --------------AVDLPAEVNNIR-EVICPDKISKCPEDTTCCLLETGSYGCCPMPKAVCCSDQKHCCPEGTTCDLIHSTCLS 242 GGPCQVD-AHCSAGHSCIFTVSGTSSCCPFPEAVACGDGHHCCPRGFHCSADGRSCEO 114

PSGSVPWVEKVPVRPIKKQKV AVTQVSSLS $\$$ DVPCNDT-AACADGTTCCKTKDGDWACCPLPEAVCCEDFVHCC PKGKKCNIAAMKCEDP 433 5 ANGVS EMAIKI PAVTVLKPKEE---------VVPCNET-VACSS GTTCCKTPEGSWACCPLPKAVCCEDHIHCC PEGTLCNVAASSCDDP 322 RSG----NNSVG----------------- AIQCPD SQFECPDFSTCCVMVDGSWGCCPMPQASCCEDRVHCCPHGAFCDLVHTRCIT 181

SCTGEPLVKOTPVQSTTTPNVIGKOKSNVPCNDTAA-CADGTTCCKTKDGDWACCPLPEAVCCEDFIHCCPHGKKCDLAAGSCDDPSGSV 522 ----TELSV SVPWMEKVSTKPIAPPP-NKKCDESSS-CPGESTCCKLSSGDWGCCPLPEAVCCEDHVHCCPHG SVCNVAAETCET VSDSA 406 TGT-HPLAKKLPAQR--TNRAVALSS-SVMCPDARSR CPDGSTCCELPSGKYGCCPMPNATCCSDHLHCCPQDTVCDLIQSKCLSKENAT 267

PWVEKVPVRPIKKOKVAVTKVSSVSSDVPCNDT AACADGTTCCKTKEGGWACCPLPEAVCCEDFTHCCPHGKKCNVAAGSCDDPSG-SVP 611 ---LRISVPMVKK----IPAVSVPS KQNCDETSSCPT GTTCCKLTSGSWACCPVPQAVCCADQEHCCPQGYTC DLAQSSCVR SGLPSMA 489 ------TDLLTK----LPAHTVG--DVKC DMEVSCPDGYTCCRLQSGAWGCCPETQAVCCEDHIHCCPAGFTCDTQKGTCEQ-GPHQVP 343

WVEKVPVHLRAG--QRSSG KVKCNATHGCPESSTCCK-NIAGEWGCCPFSOAVCCTDGEHCCPAHYKCNLS SVSCIKGDVVIPWYNKIAA 698 8 WFRKEPA-LRET--QRVEDRHMCDAHTSCPRDDTCCFI NRIGKWGCCPLPKAVCCKDGDHCCPSGYTCNEEKTSCTK GLHQIPWFTKKTA 576 WMEKAPAHLSLPDPQALKR DVPCDNVSSCPSSDTCCQ-LTSGEWGCCPIPEAVCC SDHQHCCPQGYTCVAEG-QCQPGSEIVAGLEKMPA 431

FTTPAR RVWKSSD-ELLGHEDVKCDS STSCPSGSTCCILPTGQWGCCPLVKAVCCEDHEHCC PQGYICKLELGTCEKAS------ADLSVSLTAVQ 659 RRASLSHPR----DIGCDQHTSCPVGQTCCPSLGGSWACCQLPHAVCCEDRQHCCPAGYTCNVKARSCEYEVVS----AQPATFLARSP 512

QPSVIVK DVQCGGGFSCHDGETCCPTSQTTWGCCPSPKAVCCD DMQHCCPAGYKCGPGGT-CISAGDLDWSNWVNWKLFFSKKKRALTL. 873 10 MP--- EIQCDTFTRCAHTQSCCRLADSTWACCPYTQAVCCKDMKHCCPMGY KCDPKVQGCTKSSSSTW--WNN-SL. HVG--VR DVECGEGHFC HDNQTCCRDNRQGWACCPYRQGVCCADRRHCCPAGFRCAARGTKCLPREAPRW----DAPLRDPALRQLL. 593

\section{Figure 4}

Sequence comparison of zebrafish progranulin-A and -B with human progranulin. Amino acid sequences were deduced from the cloned grna and grnb zebrafish cDNAs. Unlike human progranulin, which carries one-half and seven granulin peptide motifs, zebrafish progranulin-a and progranulin-b harbour 10 and 9 full copies of the granulin motif (boxed), and possess distinct putative signal peptides (italics). Sequences were aligned using the ClustalW method, and gaps were introduced as dashed lines for optimal alignment. Identical residues are indicated by an asterisk. Numbers on the right represent amino acid position. Human granulin motif nomenclature is listed (right).
\end{abstract}

ASgrna (see below), the observed transcript sizes were in agreement with those predicted from cloned sequences. However, many pre-mRNA transcripts of higher than expected molecular weight were identified for several family members. Only grnb demonstrated the presence of splice variants of unknown composition.

\section{Chromosomal mapping of zebrafish grns}

The chromosomal localization of each grn gene was determined using the LN54 radiation-reduced mapping panel [29]. Primers are listed in Additional File 9. grn 1 and grn2 are closely linked on linkage group (LG) 19, 5.98 centiRays $(\mathrm{CR})$ from EST clone fb47h01 in the vicinity of the simple sequence length polymorphism (SSLP) marker z6661 consistent with their physical proximity deduced from the cloning of a chimeric transcript (Figure 5). Grna is located on LG3, $9.92 \mathrm{cR}$ from SSLP marker z22516, in a region showing a clear syntenic correspondence with the chromosomal position of murine and human grn (Figure 5). In contrast, grnb maps next to SSLP marker z9325, and is $9.76 \mathrm{cR}$ from EST fc18g06, located on LG24 (data not shown), and is not part of a known block of conserved synteny with zebrafish LG3 or human chromosome 17.

\section{Assessment of zebrafish grn gene expression by RT-PCR Zebrafish grn gene expression in adult tissues}

Semi-quantitative RT-PCR analyses were performed to examine the relative expression of the individual mem- 

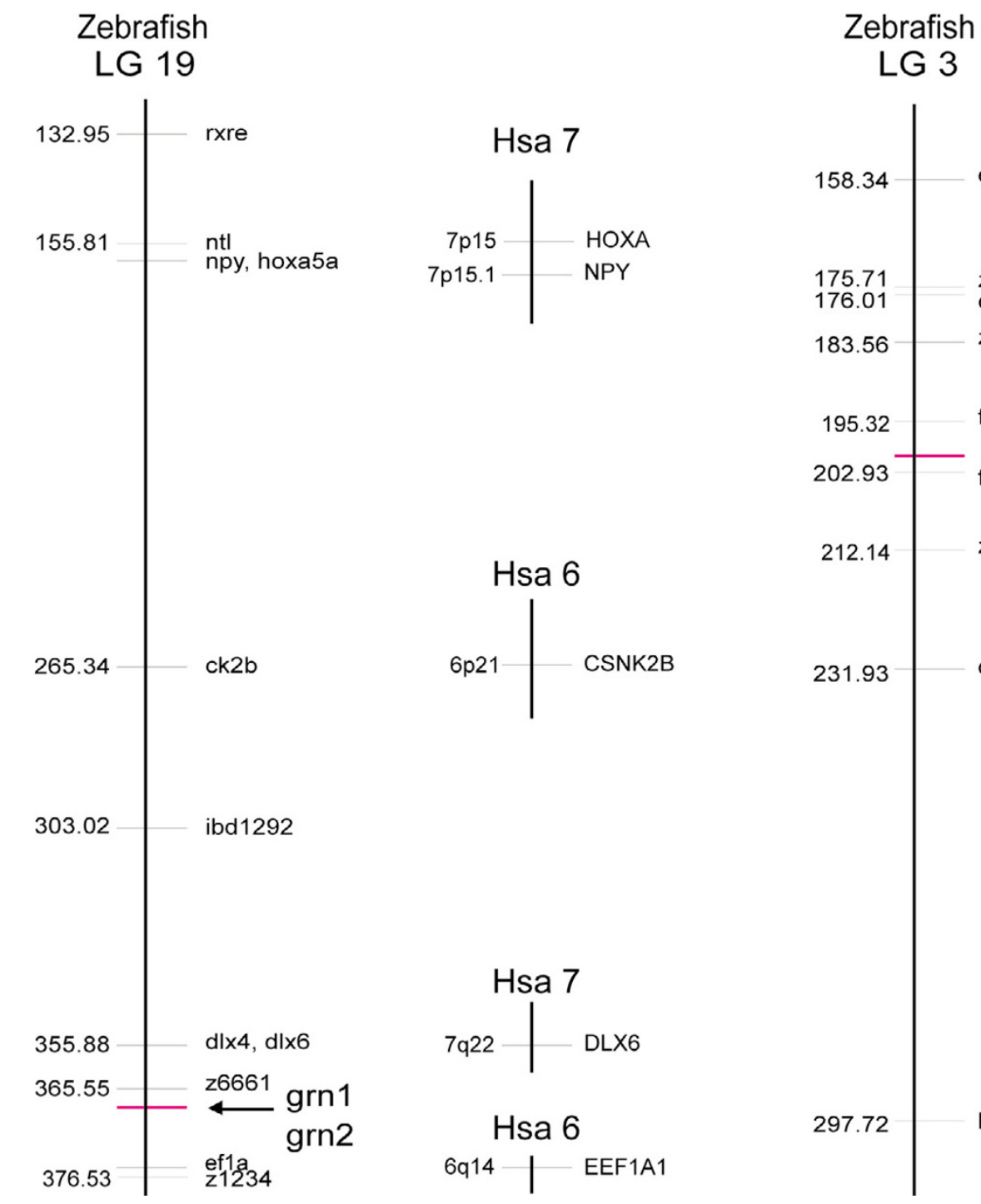
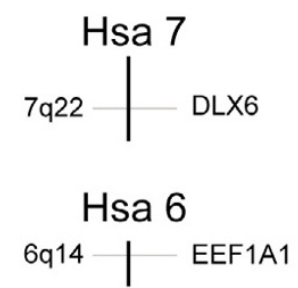
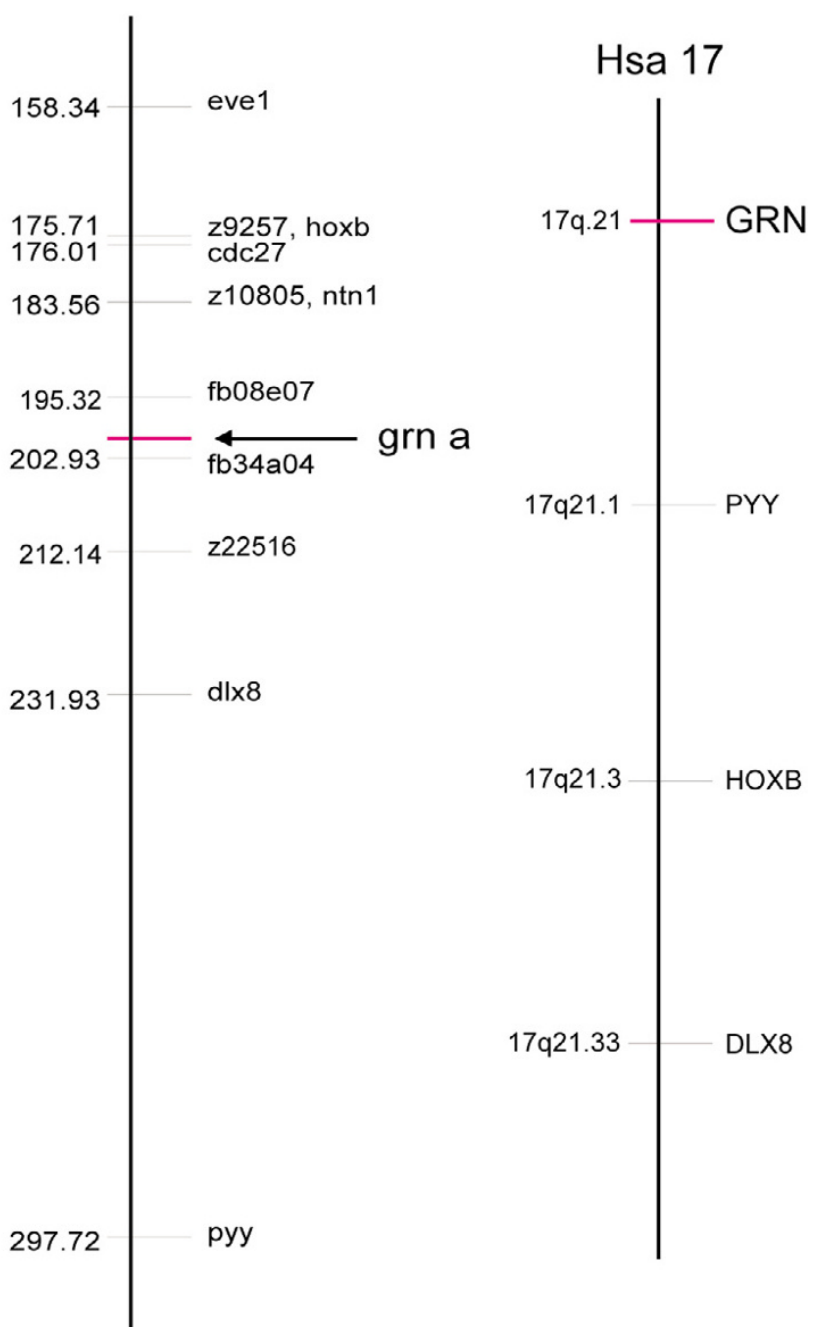

Figure 5

Chromosomal assignment of zebrafish grn genes. Zebrafish grna is located close to genes (HoxB cluster, dlx8, pyy) that form an extensive bloc of conserved synteny with human chromosome 17 (Hsa l7), indicating an orthologous relationship to human progranulin (grn). Zebrafish grnb, in contrast, maps to LG24 in a region devoid of syntenic correspondence to zebrafish LG3 or Hsal7 (data not shown). Grn I and grn2 map to LGI9, in a region that finds scattered synteny to two human chromosomes (Hsa 6 and Hsa 7). The presence of grn I and grn2 on a zebrafish chromosome bearing the HoxA cluster, npy and dlx6 genes (i.e. paralogues of genes linked to zebrafish grna and human grn), suggests that grnl or grn2 may have originated in concert with the mechanism leading to emergence of duplicated Hox clusters at the base of the vertebrate radiation. Map position on zebrafish chromosomes (LG) is presented in centiRays where I centiRay = I48 kilobases, the estimated average breakpoint frequency for the LN54 RH panel.

bers of the zebrafish grn gene family in adult tissues. A list of primers used and size of the respective amplicons is listed in Additional File 10. Both grna and grnb are expressed in all adult tissues examined, including the gills, heart, multiple visceral organs, and at modest levels in the brain (Figure 6, panel A). Comparison of the expression of the smaller zebrafish paralogues (grn1 and grn2) relative to their mutual antisense gene in selected adult zebrafish organs (Figure 6, panel B), showed that the combined expression of grn 1 and grn 2 was qualitatively similar to that of grna and grnb (Figure 6, panels B and C). In contrast to the widespread combined expression of grn 1 and grn2, low levels of ASgrn1-2 transcripts are detected in the blood and intestine (Figure 6, panel B). In agreement with the observed carp peptide expression profiles [19], the zebrafish spleen expresses grn1 only (Figure 6, panel C). 
A

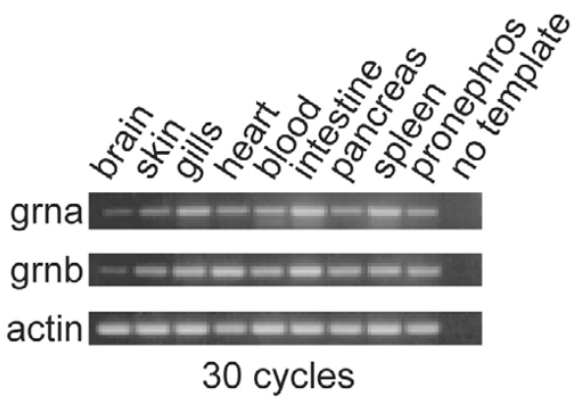

B

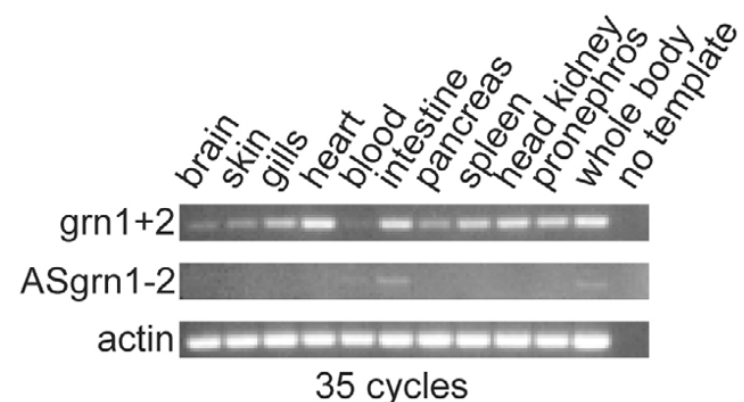

C

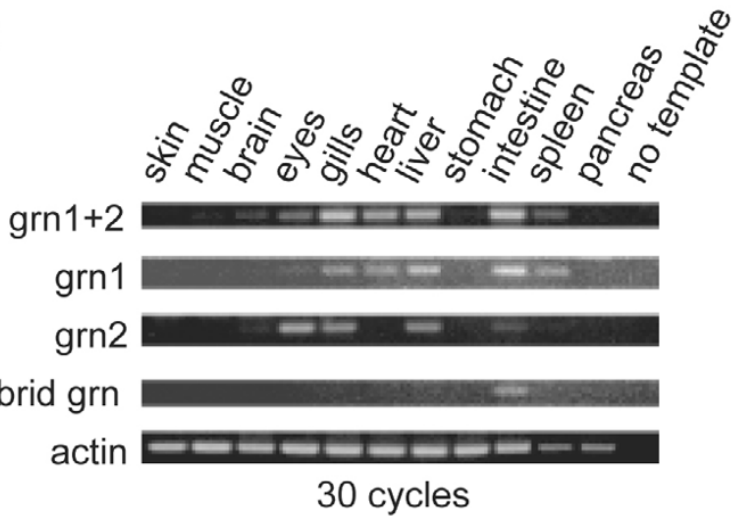

Figure 6

RT-PCR Analysis of zebrafish grns in adult tissues. Panel A: Zebrafish grna and grnb are ubiquitously expressed in various organs. Panel $B$ : A comparison of the combined expression of grn I and grn2 $(\mathrm{grnl}+2)$ relative to their antisense transcript. An increased number of cycles was used in the PCR to allow for the detection of ASgrn I-2 transcripts. Panel C: grn I, grn2 and hybrid grn are differentially regulated with the latter expressed only in the intestine. Results shown were generated using the forward and reversel primer pair (Additional File 10). Identical results were obtained using the forward and reverse2 primer pair (not shown). Hybrid grn was amplified using a grn I forward and grn2 reverse primer combination. No product was obtained using a grn2 forward and grn I reverse primer pair (not shown). Number of cycles for each reaction is indicated. Amplified PCR products were analyzed by electrophoresis next to a 100-bp DNA ladder. No template and amplification of actin mRNA were used as negative and positive controls, respectively. Similar results were obtained with two other experiments.
In tissues that display overlapping expression, grn 1 is the predominant form in the heart and intestine, while the eyes express higher levels of grn2 (Figure 6, panel C). Surprisingly, low abundance of the hybrid grn transcript, the authenticity of which was confirmed by sequencing of the amplicon, is detected exclusively in the intestine (Figure 6, panel C).

\section{Developmental expression of Zebrafish grn genes}

Since divergence in expression patterns between duplicated genes is suspected to promote their retention [18], the temporal regulation of grn gene expression between the two grn gene classes during development was investigated by RT-PCR. Transcripts for both grna and grnb are maternally provided although grnb is more abundant (Figure 7, panel A). This trend continues following commencement of zygotic transcription until mid-epiboly (shield stage) where both transcripts are present at similar levels (Figure 7, panel A). In contrast, combined grn 1 and grn2 expression is first noticeable during the late pharyngula period by 48 hours post-fertilization (hpf) (Figure 7, panel A). When using an increased number of cycling, the combined expression of grn 1 and grn 2 is detected as early as $30 \mathrm{hpf}$, while antisense transcript levels remain too low for detection using conventional ethidium bromide staining (Figure 7, panel B). Southern blot analysis demonstrates that antisense transcription occurs, albeit weakly, at $72 \mathrm{hpf}$ and becomes more evident by $120 \mathrm{hpf}$ (Figure 7, panel B).

\section{Assessment of zebrafish grn gene expression by whole mount in situ hybridization}

In order to evaluate the relationship between sense and antisense transcription, and to gain insights into the potential contributions of the four grn paralogues to development, the spatio-temporal expression of zebrafish grns was monitored by whole-mount in situ hybridization. For all stages examined, sense and antisense riboprobes for sonic hedgehog were used as experimental controls since the tissue expression of this gene is discrete and well documented [30]. No non-specific hybridization signal for sonic hedgehog was detected (data not shown). Unless stated otherwise, the respective sense riboprobes to grna and grnb did not give rise to detectable signals.

\section{Grna and grnb}

The low abundance of transcripts for grna relative to grnb revealed by RT-PCR during early embryogenesis (Figure 7, panel A) is reflected in the relative signal intensity for these transcripts by whole-mount in situ hybridization from the 4-cell to late segmentation stage (18-20 hpf) (Figure 8, panel $\mathrm{A}$ and $\mathrm{B}, \mathrm{a}-\mathrm{f}$ ). Weak, ubiquitous grna expression can first be detected by $12 \mathrm{hpf}$ ( 6 somite stage), with slightly stronger signal in the hypoblast (Figure 8, panel A, c, d). Grnb expression remains similarly ubiqui- 
tous, however defined regions of heightened expression are evident including in the hypoblast, central nervous system (CNS), optic epithelial layers, ear primordium, lateral plate mesoderm (LPM) and tailbud (Figure 8, panel $\mathrm{B}, \mathrm{C}, \mathrm{d}$ and data not shown). As the embryo matures to late segmentation stages (18-20 hpf), low levels of grna expression become confined to the eyes, tectum and tailbud (Figure 8, panel A, e, f), whereas grnb expression undergoes further regionalization within the eyes and CNS, the caudal region of the notochord and surrounding adaxial cells, and in the yolk syncytial layer (YSL) (Figure 8, panel B, e, f). During the pharyngula period (24-48 hpf) and hatching stage (72 hpf), overlapping expression patterns between grna and grnb include the pharyngeal and anterior visceral endoderm, the skin epidermis (Figure 8 , panels $\mathrm{A}$ and $\mathrm{B}, \mathrm{g}-\mathrm{i}$ ), and the pronephric tubules, albeit at modest levels (data not shown). In addition, both genes are expressed temporally within the apical ectodermal ridge (AER) between 36 and 72 hpf (Figure 8, panels $A$ and $B, h, i$ and data not shown). Divergent expression of the zebrafish co-orthologues of mammalian progranulin is also notable during this period (24-72 hpf). For instance, only grna is strongly expressed in the intermediate cell mass (ICM) caudal region, the lens and retina as well as the tectum of the $24 \mathrm{hpf}$ embryo (Figure 8, panel A, g). Grna is also uniquely expressed at low levels within the forming head vasculature and aorta at 48 hpf (Figure 8, panel A, h-i and data not shown), and exhibits relatively increased leukocytic expression from 48 to $72 \mathrm{hpf}$ (Figure 8, panel $\mathrm{A}, \mathrm{h}-\mathrm{k}$ ). In contrast to that observed for grna, grnb is expressed within the YSL and often found concentrated at the end of the yolk extension. Sustained high levels of grnb were observed within the brain at all stages examined (Figure 8, panel B, g-k) and in the swim bladder by $72 \mathrm{hpf}$ (Figure 8, panel B, k). Patterns observed during the hatching period generally persist and are accentuated in the 5 day-old larva (Figure 9, panels $\mathrm{A}$ and $\mathrm{B}$ ). In addition, grna can be detected in the epithelial lining of various visceral organs, in particular the pharynx, intestine, swim bladder and pronephric ducts (Figure 9, panel A, a). Weak staining in the dorsal aorta but not the posterior cardinal vein can sometimes be noticed in whole-mount and through sectioning of the animal (Figure 9, panel A, a). Likewise, grnb is widely expressed in the visceral region of the larval stage animal, in particular the intestine, pancreas and YSL (Figure 8 and 9 ). Weak expression is also detected for both genes in the olfactory epithelium and in the presumptive thymus as bilateral patches located caudal to the eyes (data not shown).

The sense riboprobe corresponding to grna but not grnb detects staining in the brain, intestine and pronephros at this stage (Figure 9, panel A and B, b), suggesting either non-specific hybridization or the presence of an antisense

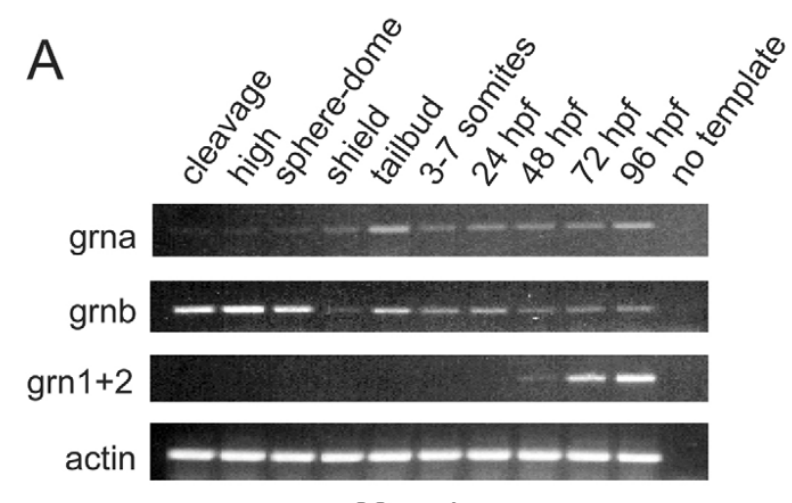

30 cycles

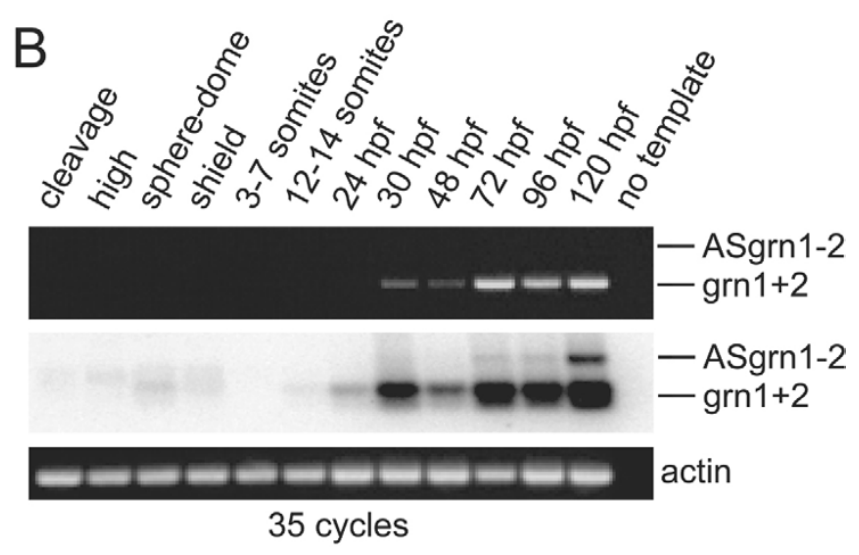

Figure 7

RT-PCR analysis of zebrafish grn expression during development. Panel A: grna and grnb transcripts are detected throughout all stages of development, whereas grn I and grn2 expression is first detected by 48 hours post-fertilization. Maternal expression of grnb is more abundant than grna. Panel B: Combined expression of grn I and grn2 relative to their antisense transcript. Ethidium bromide stain reveals the presence of sense transcription only (top). Detection of the antisense transcript is revealed by using a ${ }^{32 P}$-labelled oligo as probe that recognizes both sense and antisense amplicons after Southern transfer. Note weak expression of grnI and/or grn2 at earlier stages of development. Numbers of cycles used for the PCR are indicated. No template and actin were used as negative and positive controls, respectively. Developmental stages are as follows according to Kimmel et al. 1995: cleavage (16-cell); high (mid-blastula, 3 hpf); sphere-dome (late blastula, 4-4.3 hpf); shield (50\% epiboly, 6 hpf); tailbud ( $10 \mathrm{hpf}$ ); $3-7$ somites ( $11-12 \mathrm{hpf}$ ); I2-14 somites (14-16 hpf); early (24 hpf) and late (48 hpf) pharyngula; hatching ( $72 \mathrm{hpf}$ ); ealy larval period ( 96 and I 20 hpf). Gene specific primers and amplicon sizes are listed in Additional File 10.

transcript. This observation prompted a search for sequences deposited at GenBank corresponding to parts of the grna cDNA sequence, but in the reverse comple- 
A
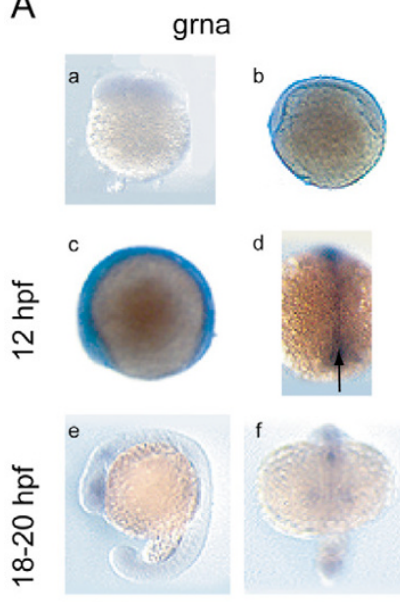

f
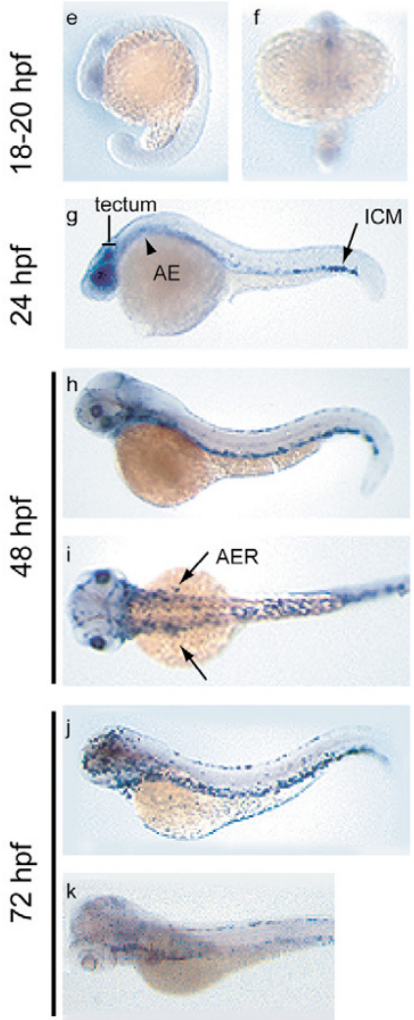

B
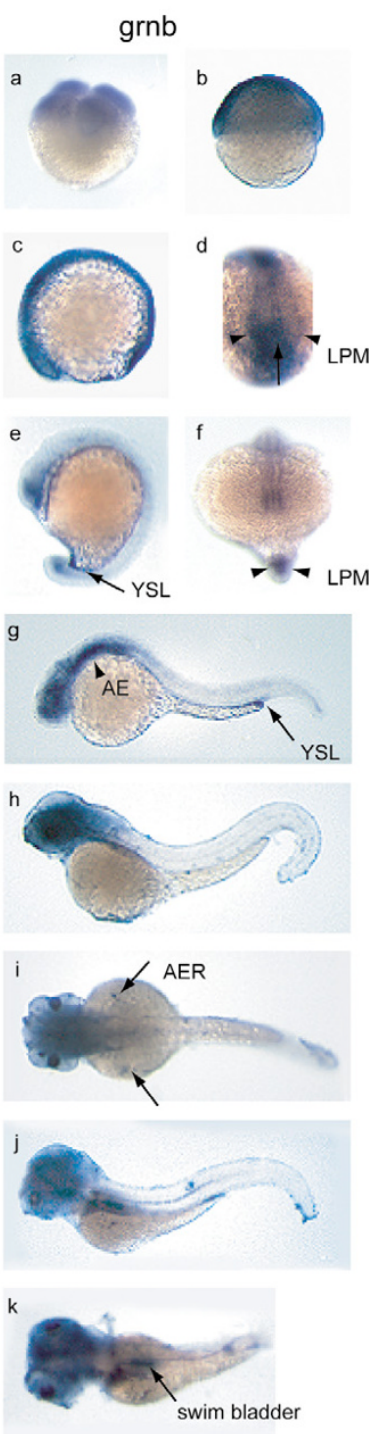

\section{Figure 8}

Developmental expression analysis of zebrafish grna and grnb mRNAs by whole mount in situ hybridization. An ontogeny of expression conducted for grna (A) and grnb (B) revealed similar expression patterns for these genes from fertilization to late segmentation stage $(a-f)$, with grna being weaker than grnb. At the 4 -cell stage (a) and $50 \%$ epiboly (b) ubiquitous expression is observed for grnb only. A lateral view of the 6-somite stage embryo (c) reveals discernable ubiquitous expression above background levels for grna, and increased grnb expression in the epithelium of the eye primordium and CNS, as well as the caudal region. In a dorsal view of the same animals (d), caudal expression in the axial mesoderm (arrow) is observed for both genes, whereas only grnb is detected in the paraxial mesoderm (arrowheads). Lateral (e) and frontal (f) views of the late somitogenesis stage embryo (18-20 hpf) show continued expression in the eye primordium, CNS and tailbud for both genes. In addition, grnb can be detected in the YSL (arrow) (e) and the adaxial cells (arrowheads) (f) flanking the axial mesoderm. At $24 \mathrm{hpf}(\mathrm{g})$, grna expression is found in the tectum and eye retina, in a diffuse pattern in the anterior endoderm (arrowhead) and in a punctuate pattern within the ventral tail region of the ICM (arrow), whereas elevated expression persists for grnb in the forebrain, midbrain and ventral hindbrain region, the eyes, as well as in the YSL, concentrated at the tip of the yolk extension (arrow). In a lateral view at $48 \mathrm{hpf}(\mathrm{h}$ ), grna expression in the ICM extends rostrally, is detected in the head vasculature, and is now apparent in the skin epithelium, whereas in a lateral view (i) both grna and grnb are transiently expressed in the AER of the pectoral fin buds (arrows). At $72 \mathrm{hpf}$ (j), grna, but not grnb, is expressed in presumed dispersed leukocytes, while in a dorsolateral view (k), grnb can be detected in the swim bladder (arrow). AE, anterior endoderm; AER, apical ectodermal ridge of the pectoral fin buds; ICM, intermediate cell mass; LPM. lateral plate mesoderm; YSL, yolk syncytial layer. 
ment orientation. Three unidirectionally cloned ESTs (accessions $\underline{\mathrm{CD} 585878}$, CD585963 and CD596001) were all revealed upon sequencing to correspond to a 914 nucleotide long cDNA sharing perfect complementarity to nucleotides 2701-3614 of the grna cDNA (Additional File 11, panel A). This sequence corresponds to the 3'UTR region of the grna gene and is not bisected by an intron at the genomic level. This precludes the conclusion that this candidate antisense transcript is not an artifact of cloning. To confirm its directionality, cDNA was synthesized for subsequent PCR amplification using a primer located within the 3'UTR exon of grna that shared complementarity to ASgrna (sense relative to grna) or using another primer that was located downstream of the cloned ASgrna sequence and within a known intron for grna (Additional File 11, panel A). This RT-PCR strategy suggests that the ASgrna deduced from cloned EST sequences may represent a splice variant, and that antisense transcription extends further in the 3'direction (Additional File 11, panel B).

\section{Grn I and grn2}

As expected from the RT-PCR data (Figure 7, panel A), expression for grn 1 or grn 2 is not detected by in situ hybridization in early development (data not shown). Expression of grn 1 is detected in the intestine and the pharyngeal region of the 3-day old animal (Figure 10, panel A) and at very low levels in the pronephros (data not shown). Grn2 does not share this expression pattern and is detected at low levels in the proctodeum (Figure 10, panel B) and is often detected in a few sporadic peripheral leukocytes (data not shown). In contrast hybrid grn is detected at relatively high levels in the proctodeum. (Figure 10, panel C). This specific expression pattern is confirmed by examining the signal obtained using sense riboprobes corresponding to grn 1 and grn 2 that, after prolonged exposure, detect weak signals from their complementary transcript (ASgrn1-2) in the pharyngeal region of these animals (Figure 10, panels D and E). Notably, the use of a sense riboprobe corresponding to hybrid grn (Figure 10, panel F) does not replicate the expression pattern observed for ASgrn1-2 (Figure 10, panel D and E).

In the 5 day-old larva, specific grn 1 expression is observed in the intestine and the swim bladder, whereas low levels can be seen in the pharynges (Figure 11A-C). Elevated levels of expression for grn2 in the brain, the pharyngeal jaw region, and in presumed peripheral leukocytes (Figure 11D-F), contrast with the pattern observed for grn1. However, both genes share higher levels of expression in the anterior or head kidneys and pronephric ducts (Figure $11 \mathrm{~B}$ and 11E). In situ hybridization of cross sections of the animals indicates that grn 1 and grn 2 expression, like that of grna and grnb, occurs in the epithelial lining of the visceral organs (data not shown). The chimeric transcript remained restricted to the proctodeum where it is expressed more abundantly than grn2, and was not detected in leukocytes at this stage (Figure 11G). In turn, the antisense gene to grn 1 and grn2 is expressed in the brain, swim bladder, and the middle segment of the intestine (Figure 11H).

\section{Discussion \\ Zebrafish progranulins: simplified molecular forms and orthologues of the mammalian gene}

The granulin peptide family was originally discovered as a component of the granule fraction of mammalian phagocytic leukocytes. A series of related cysteine-rich peptides (designated granulins A, B, C and D) were purified from extracts of human neutrophils [9]. The definition of the structure of human progranulin as a glycoprotein bearing multiple copies of the granulin motif made it apparent that granulin peptides are generated through proteolytic cleavage of this precursor within the phagolysosomal compartment of the neutrophil. This explains the roughly equimolar ratios observed for members of the granulin family that are co-packaged within this subcellular compartment. The hematopoietic tissues (spleen and head kidney) of the carp (Cyprinus carpio) were also shown to be abundant sources of three granulin-like peptides (granulins-1, -2 and -3) [19]. However, the non-stoichiometric ratios observed for carp granulins-1,-2 and -3 suggested that the granulin gene family in this teleost species may expand beyond the prototypic single grn gene found in mammals. Specifically, carp spleen contains mainly granulin-1, whereas granulins-1, -2 and -3 were found in extracts of the head kidney (Belcourt et al. 1993). To simplify the identification of teleost granulin gene family members, the zebrafish (Danio rerio) was chosen based on its usefulness as a model of vertebrate development and disease.

The presence of extra gene paralogues in teleost fish has been extensively documented, often $[15,31,32]$ but not invariably supporting $[33,34]$ the hypothesis that the actinopterygian (ray-finned) lineage underwent an additional round of genome duplication (3R) subsequent to diverging from the sarcopterygian (lobe-finned) lineage approximately 450 mya. Specific examples include the Hox clusters [16,35], the annexins [36], the claudins [37] and the Nodal-related genes squint and cyclops [38]. Further, comparative chromosomal mapping studies have shown that duplicated zebrafish genes often reside on distinct chromosomes that exhibit extensive blocs of conserved synteny with their mammalian counterpart $[39,40]$. Using this approach, it has been estimated that approximately $20 \%$ of human genes may be represented by two co-orthologues in the genome of zebrafish [41]. 
A

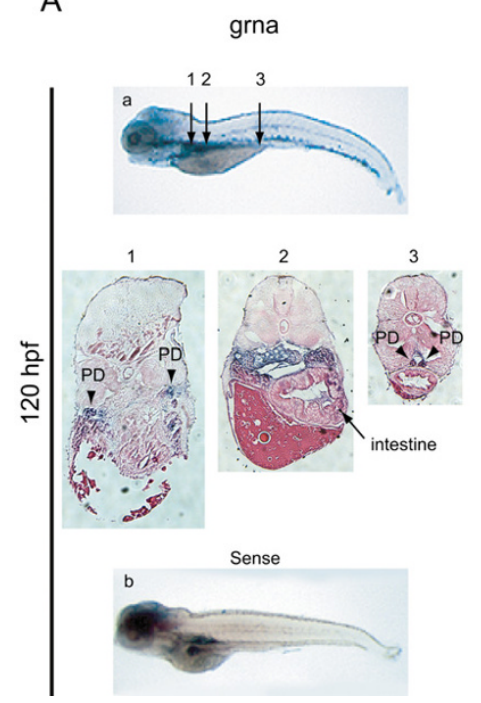

B
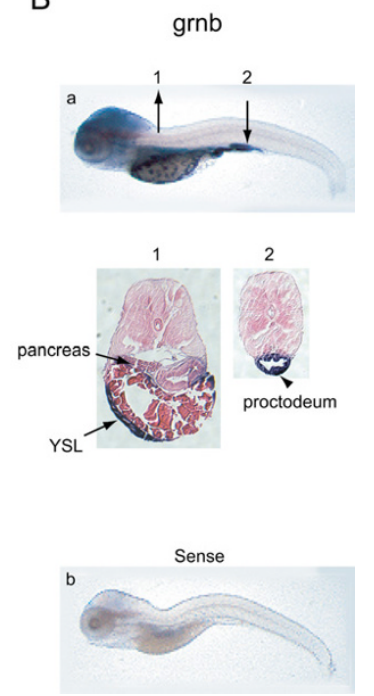

Figure 9

Expression analysis of zebrafish grna and grnb mRNAs by whole mount in situ hybridisation at 5 dpf. At $120 \mathrm{hpf}$ grna (A) exhibits widespread expression in the visceral region, including the pronephric kidneys (arrowheads in sections $I$ and 3 ) and intestine (arrow in section 2), while grnb (B) remains expressed in the YSL and pancreas (arrows in section I) and is strong in the proctodeum region of the intestine (arrow in section 2). At this stage, a hybridization signal for the sense riboprobe to grna, but not grnb, is detected in the brain, intestine and pronephric ducts (b). Numbered arrows denote the position of corresponding sections shown below (magnified I0x). PD, pronephric ducts; YSL, yolk syncytial layer.

As predicted by $3 \mathrm{R}$, we demonstrate that grns are members of an extended gene family in zebrafish. We have identified two deduced precursors, progranulins $\mathrm{A}$ and $\mathrm{B}$, harbouring 10 and 9 granulin peptide repeats respectively that bear close structural and sequence relationship to human progranulin (Figure 4). Grna was localized to a region of LG3 known to show syntenic correspondence to where human grn is found on human chromosome 17 (Figure 5). Grnb was localized to LG 24 rather than being positioned on LG12, predicted to bear synteny with LG3 [40]. Despite this apparent discrepancy, a co-orthologous relationship between grna and grnb relative to mammalian $g r n$ is supported by their sequence conservation as well as their extensive overlapping expression patterns observed during development.

Two smaller grn genes, grn 1 and grn2, each encoding one full and one amino-terminal half copies only of the granulin motif, were also characterized (Figure 2). Interestingly, a common ancestry of the smaller zebrafish grn genes with grna and grnb, and thus mammalian grn, is implied by conservation of the strict exonic organization

that these genes display. However, it is unlikely that grn1 and grn2 arose from the postulated whole genome duplication event corresponding to $3 \mathrm{R}$ since they were found to be localized in tandem on LG19 (Figure 5). It is notable that both $g r n 1$ and $g r n 2$ are linked to a Hox cluster and $d l x$ gene paralogues, similar to that observed for grna. This suggests that a smaller grn may have originated coincidentally with the duplication of a Hox-bearing chromosome in a primordial species. Evidence suggests that this putative structure would then have been retained within the teleosts but lost within the sarcopterygian line of evolution leading to mammals.

\section{Analysis of progranulin expression by RT-PCR}

The DDC model predicts that an important driving force behind the retention of duplicated genes is the devolution of an ancestral function onto the resultant pair through quantitative and qualitative changes in gene expression which, when combined, may reflect the sum of the ancestral expression pattern [18]. Thus, it was of interest to determine the extent of expression partitioning and overlap between the two grn gene classes during development by RT-PCR and to compare these patterns to those known for the mammalian counterpart.

As an initial survey of the differential expression patterns of the zebrafish grns, we conducted semi-quantitative RTPCR analyses using adult tissues and staged embryos. Similar to the well documented widespread expression pattern of human $[26,27,42]$, rat [28], mouse and guinea pig [5] grns in several tissues and cell lines of epithelial, mesenchymal, and hematopoietic origin, both grna and grnb were observed to be expressed ubiquitously in several adult zebrafish organs. In contrast, grn 1 and grn 2 exhibit a more restricted pattern of expression. It was previously shown that carp granulin-1 and granulin-2 peptides are differently distributed in the spleen and head kidneys of the carp [19]. Interestingly, RT-PCR experiments demonstrate that the expression of the homologous structures in zebrafish were similarly uncoupled at the level of mRNA, (Figure 6, panel C), reflected in a lack of detectable zebrafish grn2 expression in adult spleen. Also, grn1 appears to be the predominant form in the heart, while the eyes express higher levels of grn2. Whether the widespread pattern of expression for zebrafish grns is due in part to leukocyte entrapment in some organs, cells known to express granulins in carp [43] and goldfish [44] cannot be determined using this experimental approach.

Other notable differences in the expression of the paralogous and orthologous pairs of genes were observed. First, maternal transcripts for grna and grnb are readily detectable, with grnb showing higher abundance than grna until early somitogenesis when the two genes become expressed at similar levels (Figure 7, panel A). In contrast, 


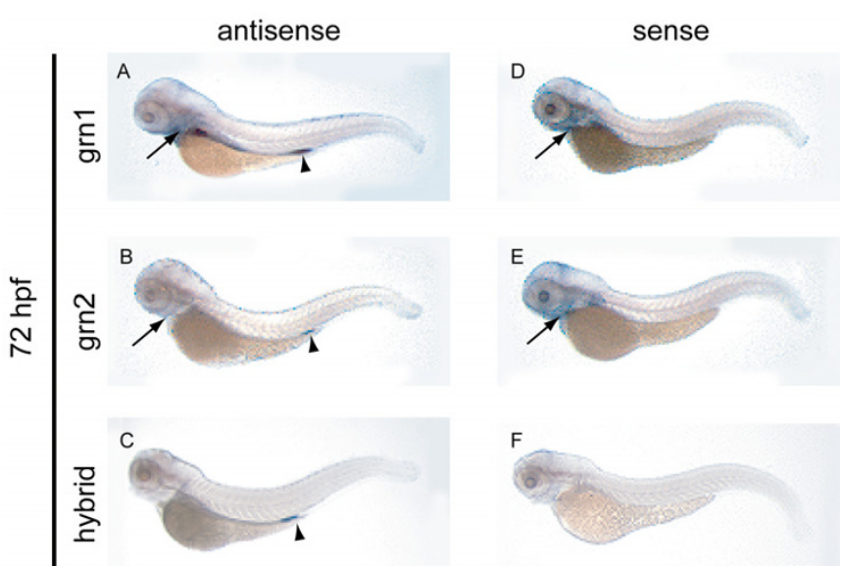

Figure 10

Expression analysis of grn I, grn2, hybrid grn, and ASgrn I-2 in the hatching stage zebrafish embryo by whole mount in situ hybridization. Panel A: grn I is expressed in the intestine and pharyngeal region (arrow), and at low levels in the pronephric ducts. Panel B: In contrast, grn2 mRNA is only weakly detected in the pharyngeal region (arrow) and the proctodeum (arrowhead), and is occasionally found in dispersed leukocytes (not shown). Panel C: The abundance of the trans-spliced product (hybrid grn) is stronger than grn2 in the proctodeum (arrowhead), but absent in the pharyngeal region. Panels D-F: The corresponding sense riboprobes to grn I and grn2, but not to hybrid grn, detect ASgrn I-2 expression in the pharyngeal region (arrows). These expression patterns were reproduced in at least three independent experiments.

although very low levels of grn 1 mRNA are also detected in the newly-fertilized egg, the combined expression of grn 1 and grn 2 only becomes detectable by $30 \mathrm{hpf}$, at a stage when most organogenesis is well advanced (Figure 7, panel A). Thus, the absence of grn 1 and grn 2 expression prior to the onset of zygotic expression argues that these genes are functionally dispensable in early embryogenesis.

\section{Analysis of progranulin expression by whole mount in situ hybridization \\ Early expression}

In order to determine possible roles for the grns during development, their spatio-temporal distribution was examined by whole mount in situ hybridization. Overall, grna and grnb expression patterns share conserved features with their murine orthologue in the early embryo. Transcripts for both zebrafish co-orthologues are maternally deposited and remain ubiquitously expressed subsequent to the onset of zygotic transcription (mid-blastula transition - 3 hpf). Similarly, in a pattern that reflects the replacement of maternal mRNAs with zygotically expressed transcripts [45], murine grn mRNA levels fall rapidly after egg fertilization, reaching negligible levels as early as the 2-cell stage, but rise again to detectable levels by the eight-cell stage [13] Notably, this precedes the morula stage and subsequent blastocyst stage when the epithelium is first formed. It is interesting to note that during zebrafish epiboly, which comprises the morphogenetic movements of the blastoderm towards the vegetal pole (dome to tailbud stage $-4.5 \mathrm{hpf}$ to $10 \mathrm{hpf}$ ), grna and grnb are still ubiquitous but more intense in the outer enveloping monolayer of cells (EVL), which ultimately will give rise to an epithelium covering the blastoderm. Expression of these grns in the EVL and later in the skin ectoderm is reminiscent of the more elevated levels of expression for grn in the apical surface of the mouse blastocyst epithelium, the trophectoderm, relative to the inner cell mass population [13].

\section{CNS}

Although several regions display increased expression of grna and grnb during brain segmentation (Figure 8, panel $\mathrm{A}$ and $\mathrm{B}, \mathrm{C}-\mathrm{f})$, regional specificity is more apparent at 24 hpf. Distinct or non-overlapping patterns observed include grna expression within the tectum and a more expansive grnb expression pattern encompassing the midbrain-hindbrain boundary, tegmentum and telencephalon (Figure 8, panel B, g). Despite the expression of both grna and grnb in the epithelial lining of the eyes and lens, transient expression within the retina and tectum is noticeable for grna only, suggesting that this orthologue may affect the development of the retinotectal projections. Similar functions may also be implied for mammalian grn, given its expression within the retinal glia during murine development [46]. Interestingly, murine grn expression is abundant throughout the central and peripheral nervous system, similar to that observed for grnb at later developmental time points within the zebrafish CNS (Figure 8 and 9). The generally unrestricted gene expression pattern suggests a role for progranulin in cell survival or proliferation or as a competence factor. Indeed, mammalian grn has been demonstrated to be a potent glial cell mitogen in vitro [47] and is consistently up-regulated in malignant human gliomas $[47,48]$. Grn2 and ASgrn1-2 but not grn1 demonstrate similar unrestricted expression within the zebrafish brain (Figure $11 \mathrm{~d}, \mathrm{~h})$. Taken together these expression patterns suggest that CNS development in the zebrafish may involve a functional interplay between the various molecular forms of granulin.

The only known functional and physiological contribution that grn gene expression is known to make during neural maturation is its involvement in the sexual differentiation of the male rat brain. It has been shown that male sexual behaviour is associated with steroid-dependent $g r n$ expression in the male neonatal hypothalamus $[49,50]$. Similar distinctions in grn gene expression were 

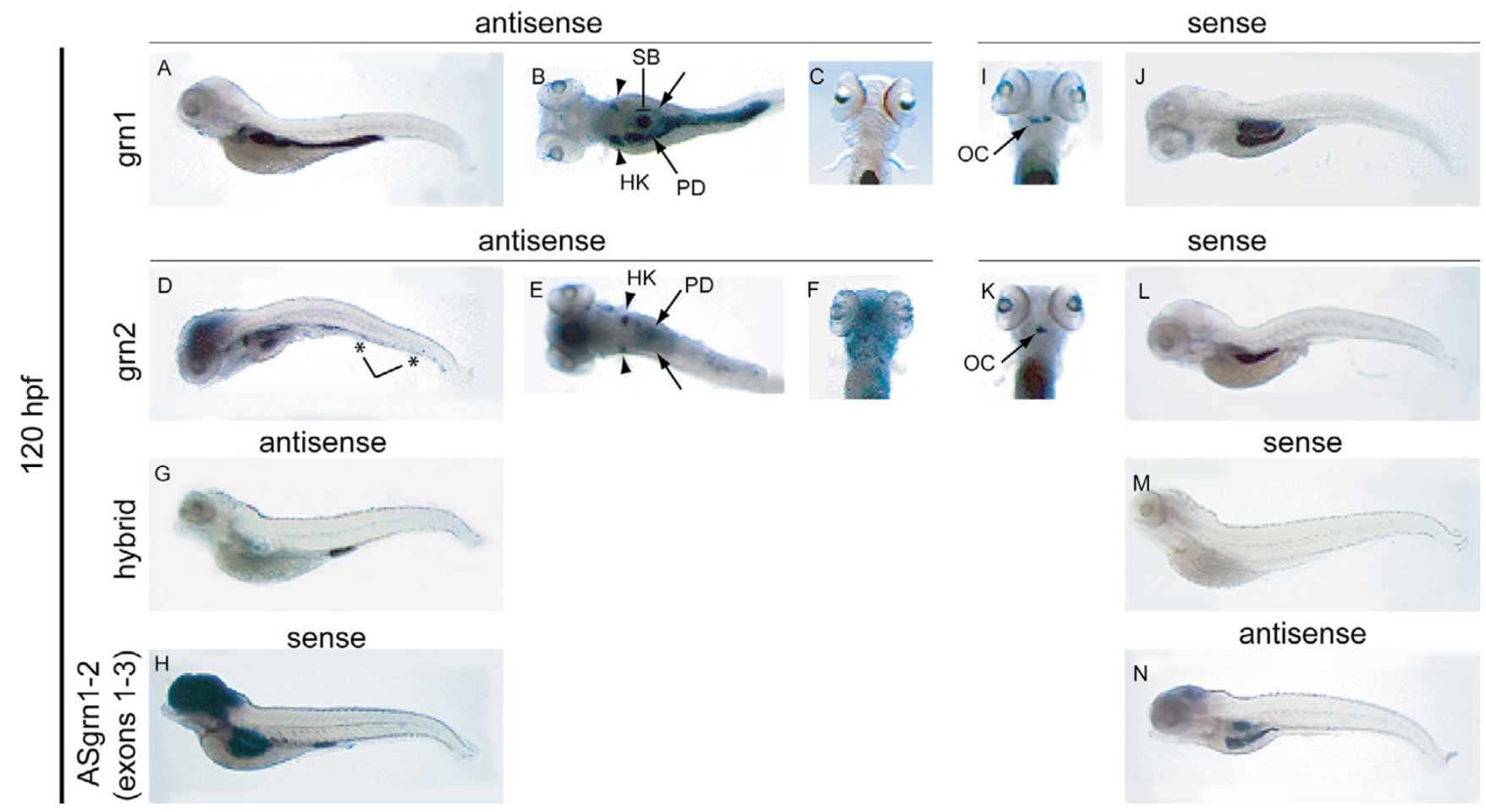

Figure I I

Expression analysis of grn I, grn2, hybrid grn, and ASgrn I-2 in the 5 day-old zebrafish larva by mRNA in situ hybridization. Panels A-C: grn I is expressed in the intestine (arrow), swim bladder, and more abundantly in the head kidneys (arrowheads) than in the pronephric ducts. Panels D-F: grn2 is expressed similarly to grn I in the head kidneys (arrowheads) and pronephric ducts, but is undetected in the intestine. In contrast, grn2 is strongly expressed in the brain and the branchial jaw region (compare $A$ with $D$, and $C$ with $F$ ), is distributed in a punctuate pattern along the ventral region of the animal in presumed myeloid progenitors (asterisks in D), and often found in randomly dispersed leukocytes (large cells in F). Panel G: Hybrid grn is found exclusively in the proctodeum. Panel $H$ : The sense riboprobe to ASgrn I-2 (devoid of the tzf sequence) recapitulates the combined expression patterns for grn I and grn2. Panels (I-N): Sense riboprobes to grn I (I,J) or grn2 (K,L), but not hybrid grn (M), show that antisense transcription occurs in the jaw region (arrows in I and $K$ ), the swim bladder, and in the mid-region of the intestine, in a pattern identical to that observed for the antisense probe corresponding to ASgrn I-2 (N). B, E: dorsal views; C, F, I, K: ventral views. For each target mRNA, the use of corresponding sense and antisense (AS) riboprobes is indicated. OC, presumed ossification center; HK, head kidney; PD, pronephric duct; SB, swim bladder.

not determined in the present study given that sexual differentiation of zebrafish gonads occurs much later and spans roughly 21-28 dpf [51].

\section{Endoderm}

The expression of the zebrafish grn gene family members also displays overlapping and distinctive patterns with respect to the endoderm and tissues derived from this germ layer. At $24 \mathrm{hpf}$ both grna and grnb can be found within the pharyngeal and foregut endoderm, whereas only the latter is located within the YSL (Figure 8, panels $\mathrm{A}$ and $\mathrm{B}, \mathrm{g}$ ). Both transcripts maintain a degree of dispersed endodermal expression until $120 \mathrm{hpf}$ where grna is located within the epithelial lining of the stomach and anterior intestine while grnb can be found within the YSL, pancreas and proctodeum (Figure 8 and 9).
Unlike the expression of grna and grnb, the paralogues grn 1, grn2 and hybrid granulin are highly abundant and for the most part restricted to pharyngeal and visceral endodermal derivatives from $72 \mathrm{hpf}$ onward. Although grn 1 and grn 2 demonstrate some endodermal tissue-specific expression, these transcripts often co-localize (Figure $11 \mathrm{a}, \mathrm{b}, \mathrm{d}, \mathrm{e})$. The restricted expression of hybrid granulin within the proctodeum is particularly striking (Figure $11 \mathrm{~g})$. Consistent with the manner in which the hybrid transcript is formed, both grn1 and grn2 are likewise expressed in the proctodeum. However, expression of these two transcripts overlaps within a large portion of the intestine and stomach where no hybrid grn transcript is found. This suggests that wherever the hybrid grn is observed, the generation of this chimeric peptide must be a highly regulated process and that a specific function is implied. 
In contrast to the observed grn distribution in zebrafish, murine grn is not detected within developing endodermal derivatives, with the exception of adult deep crypt enterocytes $[42,46]$. This suggests that mammalian dependence on grn expression may be developmentally restricted to endodermal-epithelial transitions in the gut or subsequent maintenance of this organ. Alternatively, it is possible that endodermal expression of zebrafish grns reflects a species-specific requirement.

\section{Hematopoietic tissue}

Of particular interest in regards to functional equivalence between species and functional separation between duplicated co-orthologues, is the almost complete partitioning of grn hematopoietic expression onto grna and to a much lesser extent grn2. In zebrafish, primitive hematopoiesis occurs within the anterior ICM from where nucleated erythroblasts originate and myeloid cells that can be seen circulating at $24 \mathrm{hpf}$ [52]. Hematopoietic stem cells (HSCs) are then believed to populate the dorsal aorta and yolk sac which represent the zebrafish equivalent of the mammalian aorta-gonad-mesonephros, the tissue presumed to be responsible for later definitive erythropoiesis $[53,54]$. The posterior ICM, located within the ventral tail and positive for molecular markers of all three hematopoietic lineages, may represent a secondary zebrafish HSC population or region required for HSC maturation [55]. Unlike the murine model, zebrafish definitive hematopoiesis undergoes a migratory transition from the dorsal aorta/ventral tail to the kidney (roughly $96 \mathrm{hpf}$ ), without the involvement of liver or bone marrow.

In accordance with expression patterns mentioned previously, grna only acquires distinctive tissue-specificity at 24 hpf, as is the case for its prevalence within the caudal ICM (Figure 8, panel $\mathrm{A}, \mathrm{g}-\mathrm{j}$ ), restricting its involvement to definitive hematopoietic waves. Grna can be found at low levels within the dorsal aorta at $48 \mathrm{hpf}$ (data not shown) and is highly expressed within the caudal-ventral tail region throughout all stages post-24 hpf (Figure 8 and 9), suggesting its involvement in multiple hematopoietic lineages. Significantly, sustained grna expression in this hematopoietic organ is coupled with the appearance of grna-expressing leukocytes dispersed throughout the animal with levels that peak at approximately $72 \mathrm{hpf}$ (Figure 8 , panel A, j-k). Thus grna expression in presumed granulocytes all over the body of the animal may suggest its involvement in the innate immune response of the host. Grn2 expression is also found within peripheral leukocytes, but in a sporadic pattern that is distinct from that observed for grna. Whether these differences reflect leukocyte sub-populations or activation states for these cells has not been addressed. Commensurate with the transition of ICM to kidney as the major site of hematopoiesis, grna can be found within the pronephric ducts (Figure 9) along with grn1 and grn2, which are also present in the head kidney (Figure 11, panel B and E).

Mammalian grn exhibits a similar expression pattern, particularly in neutrophils. Furthermore, murine grn can modulate the inflammatory response during wound healing, acting as both a chemokinetic factor and inhibitor of neutrophil degranulation and respiratory burst $[56,57]$. It remains to be determined whether grna supports a similar role in zebrafish.

\section{Chimeric transcription}

During the initial degenerate primer amplification of cDNAs encoding grn 1 and 2, a third CDNA was cloned and identified as sharing strict identity with portions of both grn 1 and 2. Interestingly, this granulin-hybrid showed $100 \%$ identity with exons 1 and 2 of grn-1, and with exons 3, 4 and 5 of grn-2, suggesting that this hybrid cDNA may represent a splicing of granulin-1 and 2 primary transcripts (Figure 3). Chimeric transcripts usually result from one of the following mechanisms: chromosomal translocations, transcription of neighboring genes as a single transcription unit or alternative splicing in trans. In all cases, joining of exons is predicted to occur through the recognition of canonical splice acceptor and donor sites.

A hybrid granulin structure has been previously reported through cloning of cDNA sequences in the rat [27]. Specifically, a structural splice variant of progranulin cDNA was retrieved and predicted to encode a granulin domain consisting of the amino-terminal domain of granulin-C fused to the carboxyl-terminal domain of granulin-D, consistent with the removal of an exon from the larger primary transcript $[22,28]$. The zebrafish hybrid grn described here likely originates through a mechanism other than alternative splicing from a larger primary transcript since the grn 2 gene is located 5' to the grn 1 gene (Figure 3). This topology was confirmed through the cloning and structural analysis of the partially complementary ASgrn1-2 gene. Also, we found no evidence for the presence of additional grn1-like genomic sequences located upstream of the grn2 gene, or elsewhere in the genome by Southern analysis (data not shown). In particular, no equivalent of carp grn3 was found in zebrafish. These observations suggest that the presence of hybrid grn in zebrafish likely occurs through a splicing reaction in trans between grn 1 and grn 2 pre-mRNAs, similar to the mechanism originally documented in trypanosomatids [58]. Although rare, scrambled or intergenic RNA molecules consisting of exons originating from distinct genes through a trans-splicing reaction have also been documented in vertebrates. For instance, acyl-CoA:cholesterol acyltransferase-1 (ACAT-1) and the CYP3A family of P450 cytochrome genes produce hybrid mRNA variants in 
humans [59,60]. Trans-splicing of the voltage-gated sodium channel in response to nerve growth factor stimulation, further suggests that this alternative mode of splicing can be a regulated process [61]. Indeed, there is evidence suggesting that splicing in trans may be facilitated through the recognition of regulatory elements within transcript sequences called splicing enhancers that require binding of SR proteins for activity [62]. Whether grn1 or grn2 harbour a necessary enhancer sequence that could explain the directionality of hybrid grn is currently not known. We believe the hybrid granulin represents the first example of trans-splicing with regards to the modification of a growth factor gene product.

\section{Antisense transcription}

Although the majority of deposited zebrafish EST library sequencing confirmed the existence of grn 1 and grn 2 , one particular sequence (AW777232) corresponded to the exact reverse complement to both grn 1 and grn 2 within the same exon/intron spanning region (exons 2-3 and intervening intron), and was named ASgrn1-2 accordingly. In addition, ASgrn1-2 harbours sequences for an extensively mutated tzf transposon (Tc1/mariner superfamily) in its last exon, but in the reverse complement orientation (Figure 3 and Additional File 7). Despite its polyadenylation, the lack of an ORF classifies ASgrn1-2 as a non-coding RNA. To our knowledge, ASgrn1-2 represents the first example of a single spliced transcript with antisense complementarity to two tandemly organized paralogous protein-coding genes.

The existence of ASgrn1-2 has potential implications in aspects of grn1 and grn 2 function. Classically, antisense transcripts often function as inhibitors of the expression of their associated gene, through repression of transcription or promotion of mRNA degradation. There is the possibility that ASgrn1-2 is involved in the formation of hybrid grn wherein this complementary transcript may provide a scaffold to sequester grn 1 and grn 2 mRNA transcripts within the same intracellular locale, preventing or alternatively facilitating the trans-splicing reaction.

Regarding the RT-PCR data, despite evidence of clear tissue-specific and temporal ASgrn1-2 expression in adult tissues and various developmental stages respectively, this transcript shows no clear reciprocal relationship to grn1 or grn2 (Figures 6 and 7). These expression patterns were confirmed at all stages examined using sense riboprobes for grn 1, grn2, and verified for specificity by using the corresponding sense riboprobe to hybrid grn as negative control (Figures 10 and 11, and data not shown). At $5 \mathrm{dpf}$, ASgrn1-2 is expressed in the presumed hyoid ossification center, stomach and rostral intestine, as well as swim bladder (Figure 11i-n). Although grn1 and grn2 are found within most of these tissues (particularly grn1), specific expression of ASgrn1-2 in the hyoid region undergoing osteogenesis may suggest the required down-regulation of the expression of its counterpart genes during bone development. Therefore a reciprocal relationship may exist between these genes under strict spatio-temporal regulation. Although ASgrn1-2 may mediate a degradation independent mode of gene regulation, such as alternative splicing, it clearly does not perform as a universal negative regulator of grn1 and grn2 expression.

At least one type of transcription modulation may be associated with ASgrn1-2 based on whole mount in situ hybridization analysis. There is a clear reciprocal relationship between hybrid grn expression and absence of ASgrn 1-2 transcription. At $120 \mathrm{hpf}$, hybrid grn is restricted to the distal intestine and despite the expression of its substrate transcripts within several other locales, no other grn1/grn2 rich setting is devoid of ASgrn1-2 (Figure 11). This pattern further suggests that ASgrn 1-2 expression prevents formation of the hybrid grn RNA.

The existence of ASgrn1-2 suggested that an equivalent entity may exist for one or both co-orthologues, grna and grnb. Indeed, the sense probe for grna in whole mount in situ hybridization analysis produced a consistent and reproducible signal within the intestine and pronephric ducts at 5 dpf (Figure 9, panel A, b). Several unidirectionally cloned cDNAs (accession numbers (CD585878, CD585963, and CD596001) were found to correspond to the reverse complement sequence of grna within the 3'UTR. Northern blot analysis using sense grna demonstrated a putative $4 \mathrm{~kb}$ transcript and directional cDNA synthesis followed by RT-PCR indicated this putative antisense transcript extended within the known grna intronic sequence. However, repeated attempts to clone the fulllength transcript by RACE were unsuccessful. Nevertheless, these observations provide evidence for the existence of a second naturally occurring grn complementary transcript, namely ASgrna.

\section{The granulin motif - phylogenetic and functional implications}

The granulin motif is so distinctive that it is a relatively simple matter to search the protein and nucleotide databases to obtain an impression of its phylogenetic origins. Figure 12 shows a diagrammatic representation of the nature of proteins bearing the granulin motif that can be confidently predicted through searching the CDNA, EST and/or genome sequence databases using BLAST [24]. The numbers of full granulin modules present in each putative protein is shown in cartoon form together with an impression of the phylogenetic origins of the species involved. The source of the sequence data used to predict these structures is listed as accession numbers in the legend to Figure 12. The distribution of some of the proteins bear- 
ing the granulin motif within assorted species has been partially annotated and can be viewed at various web sites $[63,64]$. With some interesting exceptions (e.g. Drosophila melanogaster), the granulin motif can be found throughout eukaryotes, both in plants and multicellular animals. In contrast there is no representation within fungi or unicellular organisms. The presence of a protein bearing the granulin motif within the slime mold (Dictyostelium discoideum) is particularly revealing since this organism is thought to be a modern representative of an amoeboid organism that was a transition species between unicellular and multicellular eukaryotes [65]. The appearance of this organism is thought to predate the divergence of plants and animals about 1.5 billion years ago. This suggests that a primordial gene bearing a single granulin motif evolved once during this transition period. The plant motif is found in just one context as a carboxyl-terminal domain of a cysteine protease that is found in many members of the viridiplantae including Arabidopsis thaliana [66] (Figure 12). Together this suggests that the founding granulin gene was represented by a single copy and composed of a single granulin motif.

In contrast to the conserved protease/granulin gene architecture found in plants, members of the animal kingdom have expanded their granulin repertoire, not via genomic or segmental duplication, but rather through tandem multiplication of the granulin ORF. For instance the Xenopus leavis and the early chordate Ciona intestinalis granulin genes encode of five and six tandemly repeated near identical granulin motifs, respectively. The variance in the number of granulin intragenic regions demonstrates a degree of plasticity during grn gene expansion. Intragenic multiplication is unlikely to have occurred as a single ancestral event; rather grn gene expansion has taken place independently in various species to varying extents. This mechanism of conserved domain repetition is not unique to grn genes. Indeed, the same type of genetic expansion is likely responsible for the repetition of immunoglobulin, EGF and lectin domains in numerous proteins.

The presence of single grn genes in protostomes is not surprising since the origins of these species predate the estimated genomic duplications within the vertebrate radiation ( $1 \mathrm{R}$ and $2 \mathrm{R}$, Figure 12 ); these large-scale events appear not to have given rise to grn gene expansion. Specifically, all members of the sarcopterygian lineage (derived from lobe-finned fish) harbour a single granulin gene of varying motif number, indicating that intragenic multiplication remains the preferred and tolerated means of granulin expansion for most vertebrates, including mammals. The same intolerance to gene duplication has not encompassed the actinopterygians (ray-finned fish), including Danio rerio and Takifugu rubripes. These species have undoubtedly expanded their granulin gene reper- toire through tandem intragenic expansion as well as genome duplication (3R, Figure 12), to yield the co-orthologues grna and grnb. Interestingly, the existence of grn1 and grn2 does not necessarily conform to these methods of gene expansion, suggesting that a specific and unidentified means of gene expansion, possibly involving ASgrn1-2, may exist.

\section{Conclusion}

Although the existence of two co-orthologues of mammalian progranulin in zebrafish is likely a result of genomewide duplication, similar genetic events have occurred within chordates prior to divergence of the ray-finned (teleost) and lobe-finned (mammalia) radiations. Indeed two rounds of genome-wide duplication are believed to have occurred [67]. More precisely, the first genome duplication probably occurred in a common ancestor of all agnathans and gnathostomes after its divergence from cephalochordates, 594 mya (million years ago). The second round is presumed to have occurred $\sim 488$ mya, within the lineage leading to jawed vertebrates after the jawless line diverged, presumably before the split between cartilaginous and bony fish. Despite this, all mammals studied thus far have retained only a single copy of the progranulin gene, whereas two rounds of genome duplication would theoretically create four progranulin genes. It is therefore interesting to consider the biological rationale behind retention of grna and grnb following the teleost genome duplication, an event not permitted within other vertebrates, in conjunction with the appearance of two extra paralogues, grn1 and grn2. Regulation by gene dosage through complementary transcription may have allowed for the retention of the smaller paralogues, while putative antisense transcription to grna may be necessary for precisely regulating the spatio-temporal activity of this growth factor.

Overall, the expression patterns of zebrafish progranulins faithfully replicate those observed for the mouse counterpart in a similar context $[42,46]$. Importantly, this indicates that the use of zebrafish will enable modeling of the contributions of progranulin activity to vertebrate development through investigating both grna and grnb. These studies will be uncomplicated by the presence of grn 1 and grn2, whose expression patterns largely do not overlap with the co-orthologues. Overall, the expression patterns for the grns indicate that these growth factors may subserve multiple functions in vivo that are consistent with the known role of their mammalian counterpart in cell growth, motility and survival.

\section{Materials and methods Tissue extraction and granulin peptide purification}

Carp (Cyprinus carpio) were purchased live at a local fish market (Waldman Plus, Montreal, QUE). Peptides from 
fish spleens were extracted using $\mathrm{C}_{18}$ Sep-Pak cartridges (Waters Canada Ltd. Mississauga, ONT) and separated using reversed-phase high-performance liquid chromatography (RP-HPLC) on a $\mathrm{C}_{18}$ Bondapak column (Waters) as previously described $[19,68]$. Column fractions were screened for cysteine content by amino acid analysis and granulin- $1 / 2$ immunoreactivity by radioimmunoassay [19]. Fractions positive for both criteria were further purified by RP-HPLC using solvents containing $0.13 \%(\mathrm{v} / \mathrm{v})$ heptafluorobutyric acid as counter-ion, and subsequently purified to homogeneity using the original solvent system containing $0.1 \%(\mathrm{v} / \mathrm{v})$ trifluoroacetic acid. Molecular weight of purified peptides was determined using a Voyager matrix-assisted laser desorption ionization time-offlight (MALDI-TOF) mass spectrometer (Perceptive Biosystems, Framingham, MA) located at the Sheldon Biotechnology Centre of McGill University.

\section{Microsequencing of carp granulin-A}

The putative carp granulin-A was alkylated according to a previously published protocol [19]. Approximately $20 \mu \mathrm{g}$ of the alkylated carp granulin-A was digested with sequencing grade chymotrypsin (Roche Diagnostics Canada, Laval, QUE) according to the manufacturer's instructions, and the resulting fragments separated by RP-HPLC on a $\mathrm{C}_{18}$ Bondapak column. Amino-terminal sequence analysis of carp granulin-A and its chymotryptic fragments was undertaken using a Procise sequencer (Applied Biosystems, Foster City, CA) located at the Sheldon Biotechnology Centre of McGill University.

\section{Fish husbandry}

Wild type zebrafish were purchased from Scientific Hatcheries (Huntington Beach, CA) and maintained on a $14 \mathrm{~h} /$ $10 \mathrm{~h}$ light/dark cycle at $28.5^{\circ} \mathrm{C}$ in a laboratory aquarium (Allantown Aquaneering, Allantown, NJ). Fish were fed twice daily, and bred as described elsewhere [69]. Embryos for developmental studies were collected from tanks and staged according to conventional criteria [70] and by hours post-fertilization (hpf).

\section{Library screening and cloning of zebrafish progranulins} The zebrafish grn 1 cDNA was cloned using a PCR strategy (Additional File 1). The carp granulin-1 amino acid sequence was used to design degenerate forward DF1 (5'GTI ATY CAY TGY GAY GC-3') and reverse DR1 (5'-CAR CAR TGR ATI CCR TC-3') and DR2 (5'-TCR CAR TGR TAI CCR TG-3') primers for use in the polymerase chain reaction (IUPAC codes are used to refer to the bases in primer sequences). The template for the initial amplification reaction was a 5'-STRETCH plus cDNA library cloned in lambda gt10 vector (Clontech BD Biosciences, Mississauga, ONT). cDNA for this library was prepared from 1month-old zebrafish using a combination of oligo-dT and random priming. $0.25 \mu \mathrm{l}$ of library (approximately $10^{8}$ $\mathrm{pfu} / \mathrm{ml}$ ) was used in a final reaction volume of $100 \mu \mathrm{l}$ for each new amplification attempt. The annealing temperature was determined empirically in order to maximize yield of product. PCR amplifications were performed with Taq DNA polymerase, unless specified otherwise, using a Hybaid thermal cycler from Bio/Can Scientific Inc. (Etobicoke, ONT). Amplified products were isolated by agarose gel electrophoresis, purified with the QIAquick Gel Extraction Kit (Qiagen Inc. Mississauga, ONT) and sequenced after cloning into TOPO pCR2.1 (Invitrogen, Carlsbad, $\mathrm{CA})$. An initial reaction using the DF1 and DR1 primer pair yielded several products. $5 \mu$ l of this reaction was subjected to re-amplification using DF1 primer in combination with the nested (anchored) DR2 primer, which revealed a product of 126-bp encoding a partial sequence for granulin-1 (Additional File 1, step 1). New grn1 primers F126 (5'-ACTGTGTGTCCAGACGG-3') and R215 (5'CCATCCCTGCAACACTG-3') were then designed based on this sequence and were used, respectively, in combination with flanking gt10 primers in order to obtain the 5 and 3'-untranslated region (UTR) CDNA sequences (Additional File 1, steps 2 and 3). Finally, the entire ORF was amplified with Pwo DNA polymerase (Roche Diagnostics), using forward F1 (5'-ATGTTCCCAGTGTTGATG-3') and reverse $\mathrm{R}$ (STOP) (5'-GCTTACAACTCCAACCCG-3') primers (Additional File 1, Step 4). This PCR was performed in a final volume of $100 \mu \mathrm{l}$, containing $0.25 \mu \mathrm{l}$ of library, $50 \mathrm{mM} \mathrm{KCl}, 10 \mathrm{mM}$ Tris- $\mathrm{HCl}$, (pH 8.8), $1.5 \mathrm{mM}$ $\mathrm{MgCl}_{2}, 0.1 \%$ Triton X-100, $0.2 \mathrm{mM}$ concentration of each dNTP, 0.5 unit of Pwo DNA polymerase, and $100 \mathrm{pmol}$ of each primer. An initial denaturation step was carried out at $94^{\circ} \mathrm{C}$ for $3 \mathrm{~min}$. Annealing temperatures of $54{ }^{\circ} \mathrm{C}, 56^{\circ} \mathrm{C}$ and $58^{\circ} \mathrm{C}$ were used sequentially for 10 cycles each. Typical denaturation, annealing, and amplification reactions were carried out at $94^{\circ} \mathrm{C}$ for $30 \mathrm{sec}, 54^{\circ} \mathrm{C}$ for $1 \mathrm{~min}$, and $68^{\circ} \mathrm{C}$ for $1 \mathrm{~min}$, respectively. A final extension step of 10 min at $72^{\circ} \mathrm{C}$ was carried out after adding 0.25 unit Taq DNA polymerase. An amplification product specific for grn 1 was sequenced on both strands. The 5'-UTR, and a portion of the 3'-UTR for grn 1, were amplified using grn 1specific primers in conjunction with a lambda gt10 primer. Distinct cDNAs encoding progranulin-2 and a chimeric progranulin were uncovered through this approach. Each transcript was confirmed through sequencing of independent amplification reactions using template cDNA derived from either adult organs or embryos of mixed stages. Following a strategy similar to that used for the isolation of zebrafish grn 1 cDNA, primers based on the purified carp granulin-A peptide sequence were designed to clone partial cDNAs for zebrafish grna and grnb, respectively (not shown). BLAST searches using the cloned sequences retrieved two unique ESTs at NCBI sharing an exact match with grna and grnb, respectively (accession numbers AW174591 and AW184435). These respective ESTs were purchased from RZPD GmbH (Hei- 


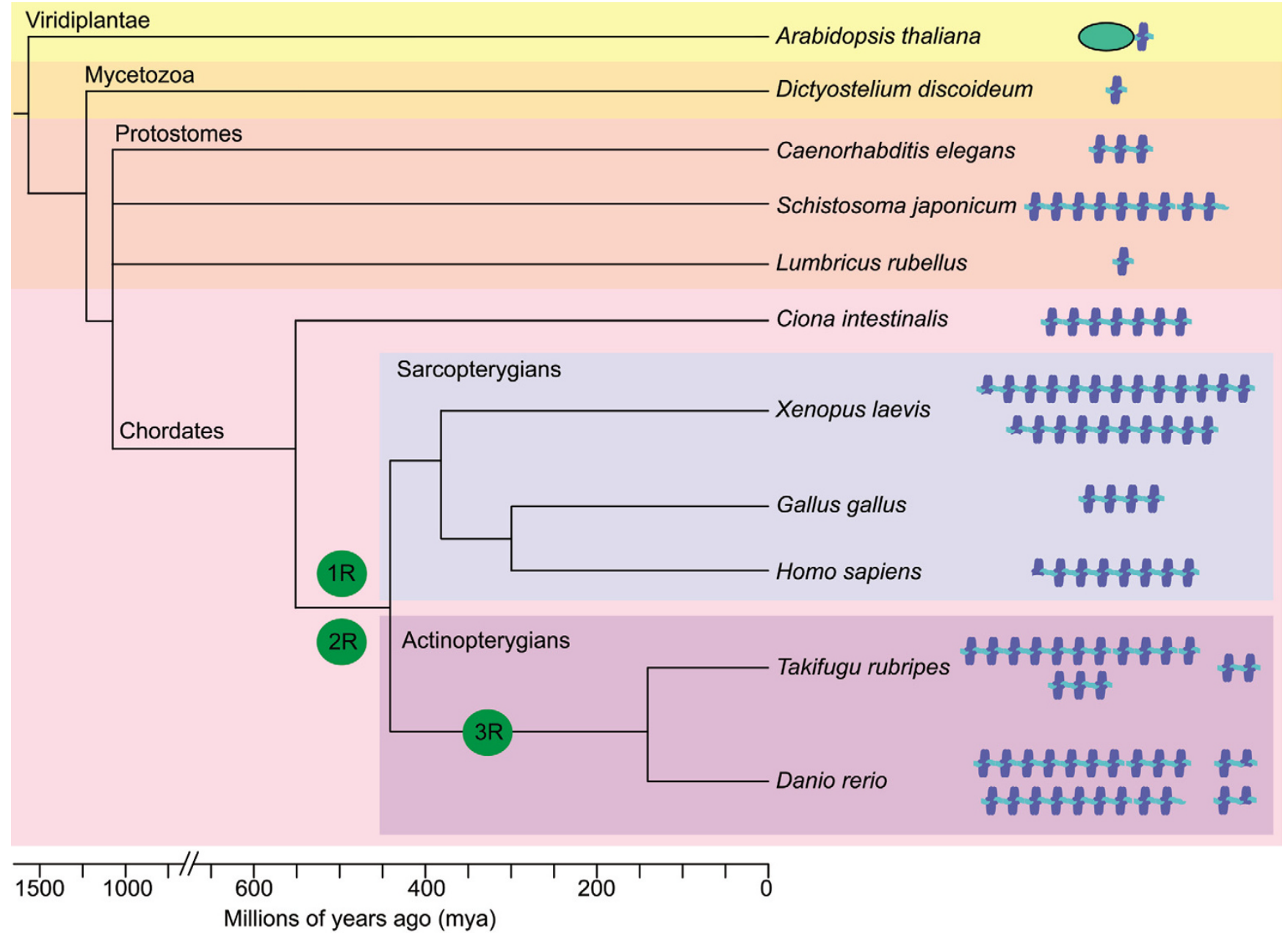

Figure 12

Diagrammatic representation of the structures and evolutionary origins of the granulin in multicelluar organisms. Evolutionary distances in millions of years derived from Hedges 2002 [77]. The estimated rounds of vertebrate genome duplication events are indicated (IR, 2R, 3R). The various progranulin structures were derived from various databases as outlined below. Land plant - (Arabidopsis thaliana) - Papain-like thiol protease bearing a carboxyl-terminal granulin domain (AAK7I3 I4); Slime mold - (Dictyostelium discoideum) - I copy progranulin from a single EST (AU26740I) Trematode worm (Schistosoma japonicum) - 9 copy progranulin built from a combination of four ESTs (AY810079, BU7902 I5, BU799560, BU77I494); Nematode worm - (Caenorhabditis elegans) - 3 copy progranulin from single EST (NM_060580) - overall architecture confirmed by genome sequence (Z8I595); Annelid worm (earthworm - Lumbricus rubellus) - I copy progranulin from a single EST (CO046860); Primitive chordate - (Sea squirt, Ciona intestinalis) - 7 copy progranulin predicted from draft genomic sequence (AABSOI000I26) and overall architecture confirmed by ESTs. Domains 2 to 7 nearly identical (BW368775, BW3 I I239). Amphibian - (Frog, Xenopus laevis) co-orthologues progranulins A and B consisting of one-half domain followed by 12 full domains and one-half domain followed by nine full domains respectively [78]. These are structurally closely related and are a result of a recent tetraploidization event 30 mya [79]. Avian (chicken - Gallus gallus) - 4 copy progranulin built from three ESTs (BM440305, BU297352 and BX265765) - overall architecture confirmed by genome sequence (LOC426606);

Human (Homo sapiens) - Progranulin composed of one half domain followed by 7 domains (UniProt entry: P28799); Teleost Takifugu rubripes - 2, 3 and II copy progranulins predicted from draft genome sequences (M000077, S002 I I8, S000 I020) with overall structures confirmed by ESTs (CA846088, CA3324II, AL842916, CA588603). Teleost - Danio rerio: from this study: co-orthologue progranulins A and B composed of 10 (NM_001001949) and 9 (AY289606) respectively; two smaller progranulins consisting of $\mathrm{I}$ and one half granulin repeats (AF273479, AF273480).

delberg, Germany; clone ID: UCDMp574E2318Q2 and UCDMp574I0223Q2) and sequenced on both strands to create a final assembly of the full-length cDNAs encoding zebrafish progranulin-A and progranulin-B. In addition to our cloning strategy, the rapid amplification of cDNA ends (RACE) was performed with the GeneRacer kit (Inv- 
itrogen, Burlington, ONT) using total RNA isolated from adult zebrafish intestine. For grn 1 and grn 2 transcripts, a reverse primer that corresponded to nucleotides 195-215 based on the cloned ORF of both transcripts (5'-CCATCCCTGCAACACTGACCC-3'), was used to perform the 5' RACE, while a forward primer corresponding to nucleotides 1-22 of each transcript (5'-ATGTTCCCAGTGTTGATGTTAC-3') was used to perform the RACE in the 3' direction. Similarly a 5' UTR sequence for grna was obtained using the map reverse primer (see below). Repeated RACE attempts in both directions for ASgrna were unsuccessful.

\section{Cloning of the zebrafish grn I gene}

A zebrafish genomic library constructed in P1 artificial chromosome (PAC) [71] and represented on filters at high-density (RZPD GmbH) was screened for the presence of the grn1 gene using standard procedures. The cDNA bearing the grn1 ORF was labeled with $\left[\alpha-{ }^{32} \mathrm{P}\right]$ dCTP by random priming using the Oligolabeling kit (Amersham Biosciences, Baie d'Urfe, QUE) for use as probe, and purified using a Sephadex G-50 column (Amersham Biosciences). Kodak X-OMAT AR film was used for autoradiography (Fisher Scientific Ltd, Whitby, ONT). Three positive clones (706K2254Q, BUSMP706K14116Q2, 706F20133Q2) were detected by autoradiography, and the first two were confirmed to carry at least part of the grn 1 gene by PCR, using the F1 (5'-ATGTTCCCAGTGTTGATG-3') and R215 (5'-CCATCCCTGCAACACTG-3') primer pair which does not discriminate between grn 1 and grn 2 , and sequencing. DNA from a positive clone (706K2254Q) was purified with the Plasmid Midi Kit (Qiagen). $1.5 \mu \mathrm{g}$ of this DNA was subjected to restriction digest with EcoRI to generate fragments suitable for cloning into pBluescript II KS (Stratagene, La Jolla, CA), and was followed by transformation in TOP 10F' electrocompetent cells (Invitrogen). Screening of colonies transferred onto nitrocellulose membranes (Xymotech, Montreal, QUE), employing the same probe used for the original library screening, was performed in the following prehybridization and hybridization conditions: $2 \times$ SSC, $0.5 \%$ SDS, $0.05 \%$ Na Pyrophosphate at $65^{\circ} \mathrm{C}$. Membranes were washed twice in $1 \times$ SSC, $0.1 \%$ SDS, $0.05 \%$ Na Pyrophosphate at $60^{\circ} \mathrm{C}$ for 15 min, followed by two washes in $0.1 \%$ SSC, $0.1 \%$ SDS, $0.05 \% \mathrm{Na}$ Pyrophosphate at $60^{\circ} \mathrm{C}$ for $10 \mathrm{~min}$. Plasmid DNA from a positive clone was purified using the high pure plasmid isolation kit (Roche Diagnostics, Laval, QUE). An insert of $\sim 9-\mathrm{kb}$ was fully sequenced and revealed the presence of the promoter region and approximately half of the grn1 gene. The remaining gene sequence was found in a $\sim 6-\mathrm{kb}$ insert clone isolated by rescreening the colony lifts with ${ }^{32} \mathrm{P}$-labeled reverse R(STOP) oligonucleotide (5'-GCTTACAACTCCAACCCG$\left.3^{\prime}\right)$ as probe. A PCR was performed using primers flanking this EcoRI site, and sequenced to confirm that the isolated $9 \mathrm{~kb}$ and $6 \mathrm{~kb}$ clones represent continuous sequences within the original PAC clone.

\section{Retrieval of antisense transcripts from NCBI}

While in the process of analyzing cloned grn1 genomic sequences (data not shown) through BLAST searches for corresponding sequences at GenBank, an EST harbouring sequences corresponding to unspliced grn 1 , but in the reverse complement orientation, was noticed. This clone (accession number AW777232) was purchased (RZPD, clone ID: DKFZp717B091Q2) and further analyzed through sequencing. A putative transcript exhibiting perfect complementarity to the 3'UTR region of zebrafish grna (ASgrna) was deduced from sequencing four unidirectionally cloned ESTs deposited at GenBank (CD585878, CD585963, CD596001 and CD588938) that originated from an oligo-dT-primed cDNA synthesis from adult kidney marrow RNA (Song et al. 2004; kindly provided by Dr. Chen, Shanghai Institute of Biological Science). Unidirectional cDNA synthesis using total RNA derived 5 day-old larvae was synthesized using a sense primer relative to the 3'UTR exon (ASgrna 2) or to a known intron (ASgrna 3) of grna (Additional File 10 and 11). These primers were then used in conjunction with the following primer (ASgrna 1) in subsequent RT-PCR to confirm antisense transcription to grna.

\section{Chromosomal assignment and syntenic analysis}

Zebrafish grns were mapped using the LN54 Radiation Hybrid Panel as previously described [29]. Primers for each gene are noted in Additional File 9. Each PCR reaction was carried in a final volume of $20 \mu \mathrm{l}$ containing 100 ng "hybrid DNA", $500 \mathrm{mM} \mathrm{KCl,} 100 \mathrm{mM}$ Tris-HCl (pH 8.3), $15 \mathrm{mM} \mathrm{MgCl}_{2}, 0.2 \mathrm{mM}$ each dNTP, 1 unit Taq DNA polymerase, and $5 \mathrm{pmol}$ of each oligo. Denaturation, annealing and amplification were performed at $94^{\circ} \mathrm{C}$ for $30 \mathrm{sec}, 55^{\circ} \mathrm{C}$ (grn 1 and grn 2 ) or $60^{\circ} \mathrm{C}$ (grna and grnb) for $30 \mathrm{sec}$, and $72^{\circ} \mathrm{C}$ for $30 \mathrm{sec}$, respectively, followed by an extension step of $7 \mathrm{~min}$ at $72^{\circ} \mathrm{C}$. To determine syntenic relationships between zebrafish and human genomes, mapped zebrafish genes flanking a given zebrafish grn gene were identified using the consolidated zebrafish maps available from ZFIN [72] and data from LocusLink [73].

\section{Gene expression profiling by RT-PCR}

Total RNA from various adult tissues and developmental stages was isolated using Trizol LS reagent (Gibco BRL, Burlington, ONT), treated with DNaseI and used in first strand synthesis using the Revert Aid H-synthesis kit (MBI Fermentas Inc. Burlington, ONT). PCR conditions used for each family member consisted of an initial denaturation at $94^{\circ} \mathrm{C}$ for $2 \mathrm{~min}$, followed by $30-40$ cycles at $94^{\circ} \mathrm{C}$ for $45 \mathrm{sec}$, gene-specific annealing temperature (Addi- 
tional File 10) for $1 \mathrm{~min}$ and extension at $72^{\circ} \mathrm{C}$ for $1 \mathrm{~min}$, with a final single cycle extension at $72^{\circ} \mathrm{C}$ for $7 \mathrm{~min}$. To discriminate between grn1, grn2 and hybrid grn, an $\left(\mathrm{NH}_{4}\right)_{2} \mathrm{SO}_{4}$ buffer with $1 \mathrm{mM} \mathrm{MgCl}{ }_{2}$ (MBI Fermentas) was used for RT-PCR in conjunction with cloned template controls (pBluescript, Clonetech) for all three forms using the same reaction parameters. Two independent reverse primers for grn1 and grn2 yielded products of expected size and hybrid grn was produced using grn 1 forward with either of the grn 2 reverse primers. All PCR products were resolved on $2 \%$ agarose gels, ethidium bromide stained and visualized on Polaroid 667 Film. The authenticity of all PCR products was confirmed by sequencing after cloning into TOPO/pCR2.1.

\section{Northern blot analysis}

Full-length mRNA transcript size was assessed for each progranulin family member by Northern analysis of polyA enriched mRNA (Micro Poly (A) Purist Small Scale Purification Kit; Ambion) derived from whole adult or $5 \mathrm{dpf}$ animals [74]. Hybridization (Ultrahyb; Ambion, Austin, TX) was carried out using non-radioactive biotin-labeled cRNA probes (Psoralen-Biotin Non-iosotopic labeling Kit; Ambion) and detected with Brightstar BioDetect Nonisotopic Detection Kit (Ambion) according to manufacturers instructions. Band size was determined using pre-labeled biotin markers (Ambion).

\section{Whole-mount mRNA in situ hybridization}

In situ hybridization for progranulin family gene expression was carried out essentially as previously described [75]. Briefly, digoxigenin-labeled RNA probes for each full-length cDNA, with the exception of ASgrn-1/2 which corresponded to exons $1-3$ only, were hybridized at $70^{\circ} \mathrm{C}$ using various developmental stages from cleavage to larval. In some cases, polyvinyl alcohol was added to the staining solution in order to minimize the occurrence of background, especially when the reaction was required to proceed for several days [76]. Stained whole-mount and sectioned embryos were mounted in glycerol and visualized under a Leica MZFLIII stereomicroscope (Richmond Hill, ONT). Pictures were taken with a Leica DC350F camera and processed with Adobe Photoshop 7.0 software.

\section{Sequence Accession Numbers}

GenBank accession numbers of all zebrafish proranulin genes and antisense transcripts described in this paper are as fellows: grn1, AF273479; grn2, AF273480; hybrid grn, AF273481; ASgrn1-2, AY289607; grna, AF375477, ASgrna, AY826190; grnb, AY289606.

\section{List of abbreviations}

AER, apical ectodermal ridge; ASgrn, antisense granulin; CNS, central nervous system; DDC, duplication-degeneration-complementation; EVL, enveloping monolayer of cells; grn, granulin; hpf, hours post-fertilization; HSC, hematopoietic stem cell; ICM, intermediate cell mass; LG, linkage group; LPM, lateral plate mesoderm; mya, million years ago; SSLP, single sequence length polymorphism; YSL, yolk syncitial layer.

\section{Authors' contributions}

Benoît Cadieux established and justified the zebrafish animal model, carried out all the molecular genetic studies and prepared the initial draft of the manuscript. Babykumari P. Chitramuthu carried out the whole-mount in situ hybridization studies and Northern blot analysis, participated in the design of the study and helped draft the manuscript, David Baranowski participated in the design of the study and helped draft the manuscript. Hugh P. J. Bennett conceived of the study, co-ordinated its design and helped draft and finalize the manuscript. All co-authors read and approved the final manuscript.

\section{Additional material}

\section{Additional File 1}

Cloning strategy for the cDNA encoding the precursor for zebrafish granulin-1. Panel A: The full-length cDNA for progranulin-1 is represented at the top of the diagram. Black rectangles represent the $O R F$, and blank rectangles represent the respective $5^{\prime}$ and $3^{\prime}$ untranslated regions. The dashed lines represent lambda phage (vector) sequences. Numbers on the left represent the sequential order of PCRs undertaken (see Materials and Methods section). Panel B: Deduced amino acid sequence for the precursor encoding granulin-1, consisting of one and one-half repeats of the granulin consensus motif. Characteristic cysteines are underlined and in bold. A predicted leader sequence is shown in italics. The full granulin-1 peptide sequence (35-91) is separated from the amino-terminal half peptide (116-147) by an intervening sequence. Stop codon is represented by *. Numbers represent amino acid position.

Click here for file

[http://www.biomedcentral.com/content/supplementary/1471-

2164-6-156-S1.pdf]

\section{Additional File 2}

Genomic architecture of the zebrafish grn1 gene. Exons (1-5) and deduced amino acid sequences are shown in uppercase letters and are boxed, while introns $(A-D)$ and flanking sequences are shown in lowercase. 5' extension of exon-1 is based on a deposited EST sequence (accession number BG884011) and 5' RACE. A potential TATA box in the promoter region is in bold and italics. The translation initiation codon (ATG) and polyadenylation signal sequence (AATAAA) are in italics. The EcoRI restriction site (gaattc) used for cloning the genomic fragments for this gene is located in intron $C$, and is underlined and bold. An identical exonic architecture was found for grn2 (data not shown).

Click here for file

[http://www.biomedcentral.com/content/supplementary/14712164-6-156-S2.pdf] 


\section{Additional File 3}

Conserved exonic organization of zebrafish grn1 and grn2 genes relative to mammalian progranulin. Like granulin repeat units found in mammalian progranulin the nucleotide sequences encoding zebrafish granulin-1 (and granulin-2, not shown here) is derived from the joining of two spliced exons with phase 0 boundaries. This characteristic splicing occurs at nucleotide positions corresponding to four amino acids after cysteine 6 within the amino-terminal region (exon 2), and two amino acids before cysteine 7 in the carboxyl-terminal end (exon 3 ). The relative sizes of exons (1-5) and introns $(A-D)$ is indicated.

Click here for file

[http://www.biomedcentral.com/content/supplementary/14712164-6-156-S3.pdf]

\section{Additional File 4}

Splice junctions of the zebrafish grn1, grn2 and the non-protein coding ASgrn1-2 genes. The consensus sequence for splice donor and acceptor sites is shown on the top line (Breathnach and Chambon, 1981). The nucleotide sequences surrounding the sites for introns $A-D$ for the respective grn 1 and grn 2 genes, as well as for introns A-C of the ASgrn1-2 gene, are shown. Exons are in uppercase, introns in lowercase. Phase of introns interrupting open reading frames are indicated.

Click here for file

[http://www.biomedcentral.com/content/supplementary/14712164-6-156-S4.pdf]

\section{Additional File 5}

Cloning of a chimeric transcript that encodes a hybrid progranulin. Panel A: Nucleotide sequence alignment of the ORFs for progranulin-1, chimeric progranulin, and progranulin-2. RACE confirmed that the cloned cDNAs possess identical 5' and 3' untranslated regions (not shown). After verifying the cDNA nucleotide sequences for grn 1 and grn2 with corresponding exonic segments (boxed), it was found that for the chimeric (hybrid grn) transcript, all except one nucleotide substitution ( $T$ instead of $C$, exon 4), are conserved and non-randomly distributed among corresponding exonic sequences of either the grn1 (highlighted in blue, exon 2) or grn2 (highlighted in orange, exons 3-5) genes. The translation initiation (ATG) and termination (TAA) codons are in bold. Arrows indicate location of primers used for RT-PCR analyses (bold; see Additional File 10). Panel B: Sequence alignment of the deduced translated sequences for progranulin-1, progranulin-2, and hybrid progranulin. The candidate chimeric transcript consists of the amino-terminal portion of grn1 (exons 1 and 2) and of the carboxyl-terminal portion of progranulin2 (exons 3 to 5). The position of introns (A-D) located in the respective grn1 and grn2 genes are indicated by arrowheads. The granulin-1 peptide and amino-terminal half-domain are underlined.

Click here for file

[http://www.biomedcentral.com/content/supplementary/14712164-6-156-S5.pdf]

\section{Additional File 6}

Nucleotide sequence of a transcript antisense to zebrafish grn1 and grn2 genes. The 1989 nucleotide ASgrn1-2 cDNA is encoded on four exons (boxed) and bears a polyadenylation signal (AATAAA) (bold and italics). Sequences corresponding to exons 2 and 3 of ASgrn1-2 are complementary to regions encompassing the second and third exons (uppercase and bold) and intronic sequences (lowercase) of the grn 1 and grn 2 genes, respectively. The nucleotide sequence of exon 4 corresponds to a mutated transposase gene of the tzf transposon sub-class of the Tc1/mariner superfamily of mobile elements, but in the reverse complement orientation. ASgrn1-2 is a non-protein coding RNA based on the absence of a predictable open reading frame.

Click here for file

[http://www.biomedcentral.com/content/supplementary/14712164-6-156-S6.pdf]

\section{Additional File 7}

The genomic region encompassing exon 4 of the ASgrn1-2 gene corresponds to a defective transposon of the tzf family, in the reverse complement orientation. A consensus 1621 nucleotide sequence for the tzf transposon, deduced by the majority rule from aligned sequences obtained from GenBank EST entries (ㄴ1226-U51230) and published data (Lam et al. 1996), is shown on top in uppercase, with the characteristic 200 nucleotides inverted repeats (underlined) and terminal TA dinucleotides (bold). The translation initiation (ATG) and termination (TGA) codons of the transposase gene are in bold and boxed. The reverse complement sequence of the region encompassing exon 4 of the ASgrn1-2 gene (boxed) is aligned underneath the transposon sequence, with mismatches represented in lowercase. Deletions (-) and insertions (+) relative to the intact transposon sequence that have occurred within exon 4 and flanking intronic sequences of the ASgrn1-2 gene, rendering this mobile element inactive, are indicated.

Click here for file

[http://www.biomedcentral.com/content/supplementary/14712164-6-156-S7.pdf]

\section{Additional File 8}

Northern analysis of zebrafish grn sense and antisense transcripts. Northern blot analyses using strand-specific cRNA probes were performed to confirm the size of cloned cDNA sequences and resolve the issue of antisense transcription to grna. Panel A: grna is expressed as a predominant transcript of $3.7 \mathrm{~kb}$ in size, consistent with cloned sequences (3649 bp), and can also be found as a larger transcript exceeding $6 \mathrm{~kb}$. Panel B: Four grnb transcripts of approximately $1.5 \mathrm{~kb}, 1.8 \mathrm{~kb}, 2.9 \mathrm{~kb}$ and $5.2 \mathrm{~kb}$ in size, respectively, are detected. Note that the $2.9 \mathrm{~kb}$ band, showing strongest intensity, is in agreement with cloned sequences for this mRNA (2820 bp). Panel C: A riboprobe targeting ASgrna detects a faint transcript of approximately $4 \mathrm{~kb}$ in size, suggesting that the cloned sequences for this antisense transcript (914 bp) may represent a splice variant. Panel D: The grn1 transcript is approximately $0.8 \mathrm{~kb}$ in size, as expected from cloned sequences (see Additional Files 2 and 3), but also expressed at lower levels as a larger transcript of approximately $1.7 \mathrm{~kb}$. Panel E: Unlike grn 1, grn2 is not expressed as a transcript other than $0.8 \mathrm{~kb}$ in size. Panel F: A band of low intensity can be detected at $0.8 \mathrm{~kb}$ for the hybrid grn RNA, suggesting that no cross-hybridization occurs with grn1 and grn2 $m R N A s$ using this riboprobe. The hybrid grn riboprobe also detects a band of approximately $1.7 \mathrm{~kb}$ similar in intensity to that seen for grn1. Panel G: The overall abundance of the ASgrn1-2 transcript is too low for detection. A-C: $15 \mu \mathrm{g}$ poly-A enriched RNA; D-G: $15 \mu \mathrm{g}$ total RNA, each derived from a whole adult.

Click here for file

[http://www.biomedcentral.com/content/supplementary/14712164-6-156-S8.pdf] 


\section{Additional File 9}

Primers used in the linkage group assignment of the zebrafish progranulin genes. Before applying to the LN 54 mapping panel, conditions for each primer combination were optimized by PCR with the use of zebrafish genomic DNA derived from the $A B$ wild type strain. The authenticity and specificity of each amplicon was verified by sequencing after cloning into the pCRII plasmid. For the assignment to zebrafish linkage groups, each PCR amplification experiment was performed at least twice. Click here for file

[http://www.biomedcentral.com/content/supplementary/1471 2164-6-156-S9.pdf]

\section{Additional File 10}

Sequences of primers and predicted sizes of PCR amplicons. All primer pairs are located on consecutive exons of their corresponding gene. Click here for file

[http://www.biomedcentral.com/content/supplementary/14712164-6-156-S10.pdf]

\section{Additional File 11}

Partial characterization of a putative antisense transcript to zebrafish grna. Panel A: Schematic representation of the location of ASgrna, a unidirectionally cloned transcript sharing complementarity with part of the last exon of the zebrafish grna gene. The relative position of primers used for cDNA synthesis (primers 2 and 3, respectively) and subsequent RTPCR are indicated (see Materials and Methods). Panel B: RT-PCR analysis of cDNA synthesized using either primer 2 or primer 3, respectively, with (+) or without (-) reverse-transcriptase. Primers depicted in A are used in the following combination for the RT-PCR: pair 1/2 (342 bp prod$u c t$ ) and pair 1/3 (478 bp product). The presence of an amplicon for both primer pairs using each $+R T C D N A$ template, but not using template derived from-RT reactions, indicates that ASgrna extends in the 3 'end direction and overlaps with intronic sequences of grna. Positive controls for PCR conditions using genomic DNA as template, as well as a no template control are indicated, respectively. The authenticity of the amplicons was determined through sequencing. Each step of the experimental procedure was performed twice.

Click here for file

[http://www.biomedcentral.com/content/supplementary/14712164-6-156-S11.pdf]

\section{Acknowledgements}

This work was supported by an operating grant (MOP-53I05) from the Canadian Institute of Health Research. We are indebted to Drs. Marc Ekker and Marie-Andrée Akimenko (University of Ottawa) for their hospitality and for sharing their technical expertise with the whole mount in situ hybridisation technique during the initial phases of this project, as well as for performing the linkage analyses on the LN54 mapping panel. We also thank Ms. Jo-Ann Bader (Molecular Oncology Group, Royal Victoria Hospital, Montreal) for providing assistance with sectioning and staining tissue sections.

\section{References}

I. Bateman A, Bennett HP: Granulins: the structure and function of an emerging family of growth factors. J Endocrinol 1998, I58(2): I45-I5I.

2. He Z, Bateman A: Progranulin (granulin-epithelin precursor, PC-cell-derived growth factor, acrogranin) mediates tissue repair and tumorigenesis. J Mol Med 2003, 8I ( I 0):600-6I2.

3. Ong $\mathrm{CH}$, Bateman A: Progranulin (granulin-epithelin precursor, PC-cell derived growth factor, acrogranin) in proliferation and tumorigenesis. Histol Histopathol 2003, I 8(4): I 275-I 288.
4. Serrero G: Autocrine growth factor revisited: PC-cell-derived growth factor (progranulin), a critical player in breast cancer tumorigenesis. Biochem Biophys Res Commun 2003 , 308(3):409-4I3.

5. Baba T, Hoff HB, Nemoto H, Lee H, Orth J, Arai Y, Gerton GL: Acrogranin, an acrosomal cysteine-rich glycoprotein, is the precursor of the growth-modulating peptides, granulins, and epithelins, and is expressed in somatic as well as male germ cells. Mol Reprod Dev 1993, 34(3):233-243.

6. Zhou J, Gao G, Crabb JW, Serrero G: Purification of an autocrine growth factor homologous with mouse epithelin precursor from a highly tumorigenic cell line. I Biol Chem 1993, 268(15): 10863-10869.

7. He Z, Bateman A: Progranulin gene expression regulates epithelial cell growth and promotes tumor growth in vivo. Cancer Res 1999, 59(13):3222-3229.

8. Xu SQ, Tang D, Chamberlain S, Pronk G, Masiarz FR, Kaur S, Prisco $M$, Zanocco-Marani T, Baserga R: The granulin/epithelin precursor abrogates the requirement for the insulin-like growth factor I receptor for growth in vitro. I Biol Chem 1998, 273(32):20078-20083.

9. Bateman A, Belcourt D, Bennett H, Lazure C, Solomon S: Granulins, a novel class of peptide from leukocytes. Biochem Biophys Res Commun 1990, I73(3): I|61-1168.

10. Shoyab M, McDonald VL, Byles C, Todaro GJ, Plowman GD: Epithelins I and 2: isolation and characterization of two cysteinerich growth-modulating proteins. Proc Natl Acad Sci U S A 1990 , 87(20):7912-7916.

II. Sparro G, Galdenzi G, Eleuteri AM, Angeletti M, Schroeder W, Fioretti $E$ : Isolation and $\mathbf{N}$-terminal sequence of multiple forms of granulins in human urine. Protein Expr Purif 1997, I0(2): 169-174.

12. Hrabal R, Chen Z, James S, Bennett HP, Ni F: The hairpin stack fold, a novel protein architecture for a new family of protein growth factors. Nat Struct Biol 1996, 3(9):747-752.

13. Diaz-Cueto L, Stein P, Jacobs A, Schultz RM, Gerton GL: Modulation of mouse preimplantation embryo development by acrogranin (epithelin/granulin precursor). Dev Biol 2000, 2 I 7(2):406-4I8.

14. Qin J, Diaz-Cueto L, Schwarze JE, Takahashi Y, Imai M, Isuzugawa K, Yamamoto S, Chang KT, Gerton GL, Imakawa K: Effects of Progranulin on Blastocyst Hatching and Subsequent Adhesion and Outgrowth in the Mouse. Biol Reprod 2005

15. Taylor JS, Braasch I, Frickey T, Meyer A, Van de Peer Y: Genome duplication, a trait shared by 22000 species of ray-finned fish. Genome Res 2003, I3(3):382-390.

16. Amores A, Force A, Yan YL, Joly L, Amemiya C, Fritz A, Ho RK, Langeland J, Prince V, Wang YL, Westerfield M, Ekker M, Postlethwait $\mathrm{JH}$ : Zebrafish hox clusters and vertebrate genome evolution. Science 1998, 282(5394): |7| I-17|4.

17. Postlethwait JH, Yan YL, Gates MA, Horne S, Amores A, Brownlie A Donovan A, Egan ES, Force A, Gong Z, Goutel C, Fritz A, Kelsh R, Knapik E, Liao E, Paw B, Ransom D, Singer A, Thomson M, Abduljabbar TS, Yelick P, Beier D, Joly JS, Larhammar D, Rosa F, Westerfield $\mathrm{M}$, Zon LI, Johnson SL, Talbot WS: Vertebrate genome evolution and the zebrafish gene map. Nat Genet I998, I 8(4):345-349.

18. Force A, Lynch M, Pickett FB, Amores A, Yan YL, Postlethwait J: Preservation of duplicate genes by complementary, degenerative mutations. Genetics 1999, I 5 I (4): |53 I-I545

19. Belcourt DR, Lazure C, Bennett HP: Isolation and primary structure of the three major forms of granulin-like peptides from hematopoietic tissues of a teleost fish (Cyprinus carpio). J Biol Chem 1993, 268(13):9230-9237.

20. David L, Blum S, Feldman MW, Lavi U, Hillel J: Recent duplication of the common carp (Cyprinus carpio L.) genome as revealed by analyses of microsatellite loci. Mol Biol Evol 2003, 20(9): | $425-1434$

21. Schreeb KH, Groth G, Sachsse W, Freundt KJ: The karyotype of the zebrafish (Brachydanio rerio). J Exp Anim Sci 1993 , 36(I):27-3I.

22. Bhandari V, Bateman A: Structure and chromosomal location of the human granulin gene. Biochem Biophys Res Commun 1992, I 88(I):57-63.

23. Baba T, Nemoto H, Watanabe K, Arai Y, Gerton GL: Exon/intron organization of the gene encoding the mouse epithelin/granulin precursor (acrogranin). FEBS Lett 1993, 322(2):89-94. 
24. Altschul SF, Gish W, Miller W, Myers EW, Lipman : Basic local alignment search tool. J Mol Biol 1990, 21 5:403-4I0.

25. Lam WL, Lee TS, Gilbert W: Active transposition in zebrafish. In Proc Natl Acad Sci USA Volume 93. ; 1996: 10870-10875.

26. Bhandari $V$, Palfree $R G$, Bateman $A$ : Isolation and sequence of the granulin precursor cDNA from human bone marrow reveals tandem cysteine-rich granulin domains. Proc Natl Acad Sci U S A 1992, 89(5): $1715-1719$.

27. Plowman GD, Green JM, Neubauer MG, Buckley SD, McDonald VL, Todaro GJ, Shoyab M: The epithelin precursor encodes two proteins with opposing activities on epithelial cell growth. J Biol Chem 1992, 267( ( 8): 1 3073-13078.

28. Bhandari V, Giaid A, Bateman A: The complementary deoxyribonucleic acid sequence, tissue distribution, and cellular localization of the rat granulin precursor. Endocrinology 1993, I33(6):2682-2689.

29. Hukriede NA, Joly L, Tsang M, Miles J, Tellis P, Epstein JA, Barbazuk WB, Li FN, Paw B, Postlethwait JH, Hudson TJ, Zon LI, McPherson JD, Chevrette M, Dawid IB, Johnson SL, Ekker M: Radiation hybrid mapping of the zebrafish genome. Proc Natl Acad Sci U S A 1999, 96(17):9745-9750.

30. Concordet JP, Lewis KE, Moore JW, Goodrich LV, Johnson RL, Scott MP, Ingham PW: Spatial regulation of a zebrafish patched homologue reflects the roles of sonic hedgehog and protein kinase $\mathbf{A}$ in neural tube and somite patterning. Development 1996, I 22(9):2835-2846.

3I. Jaillon O, Aury JM, Brunet F, Petit JL, Stange-Thomann N, Mauceli E, Bouneau L, Fischer C, Ozouf-Costaz C, Bernot A, Nicaud S, Jaffe D, Fisher S, Lutfalla G, Dossat C, Segurens B, Dasilva C, Salanoubat M, Levy M, Boudet N, Castellano S, Anthouard V, Jubin C, Castelli V, Katinka M, Vacherie B, Biemont C, Skalli Z, Cattolico L, Poulain J, De Berardinis V, Cruaud C, Duprat S, Brottier P, Coutanceau JP, Gouzy J, Parra G, Lardier G, Chapple C, McKernan KJ, McEwan P, Bosak S, Kellis M, Volff JN, Guigo R, Zody MC, Mesirov J, Lindblad-Toh K, Birren B, Nusbaum C, Kahn D, Robinson-Rechavi M, Laudet V, Schachter V, Quetier F, Saurin W, Scarpelli C, Wincker P, Lander ES, Weissenbach J, Roest Crollius H: Genome duplication in the teleost fish Tetraodon nigroviridis reveals the early vertebrate protokaryotype. Nature 2004, 43 I (70 I I):946-957.

32. Naruse K, Tanaka M, Mita K, Shima A, Postlethwait J, Mitani H: A medaka gene map: the trace of ancestral vertebrate protochromosomes revealed by comparative gene mapping. Genome Res 2004, I4(5):820-828.

33. Robinson-Rechavi M, Marchand $O$, Escriva $H$, Bardet PL, Zelus $D$, Hughes S, Laudet V: Euteleost fish genomes are characterized by expansion of gene families. Genome Res 200I, I I(5):78I-788.

34. Niimura $Y, N$ ei $M$ : Comparative evolutionary analysis of olfactory receptor gene clusters between humans and mice. Gene 2005, 346:| $3-2 \mid$.

35. Amores A, Suzuki T, Yan YL, Pomeroy J, Singer A, Amemiya C, Postlethwait JH: Developmental roles of pufferfish Hox clusters and genome evolution in ray-fin fish. Genome Res 2004, I4(I): $1-10$.

36. Farber SA, De Rose RA, Olson ES, Halpern ME: The zebrafish annexin gene family. Genome Res 2003, I3(6A): 1082-1096.

37. Loh YH, Christoffels A, Brenner S, Hunziker W, Venkatesh B: Extensive expansion of the claudin gene family in the teleost fish, Fugu rubripes. Genome Res 2004, I 4(7): 1248-1257.

38. Feldman B, Gates MA, Egan ES, Dougan ST, Rennebeck G, Sirotkin HI, Schier AF, Talbot WS: Zebrafish organizer development and germ-layer formation require nodal-related signals. Nature 1998, 395(6698): $181-185$.

39. Barbazuk WB, Korf I, Kadavi C, Heyen J, Tate S, Wun E, Bedell JA, McPherson JD, Johnson SL: The syntenic relationship of the zebrafish and human genomes. Genome Res 2000, I0(9): $1351-1358$.

40. Woods IG, Kelly PD, Chu F, Ngo-Hazelett P, Yan YL, Huang H, Postlethwait JH, Talbot WS: A comparative map of the zebrafish genome. Genome Res 2000, I0(I 2):1903-1914.

4I. Postlethwait JH, Woods IG, Ngo-Hazelett P, Yan YL, Kelly PD, Chu F, Huang $H$, Hill-Force A, Talbot WS: Zebrafish comparative genomics and the origins of vertebrate chromosomes. Genome Res 2000, I0(12): 1890-1902.

42. Daniel R, He Z, Carmichael KP, Halper J, Bateman A: Cellular localization of gene expression for progranulin. J Histochem Cytochem 2000, 48(7):999-1009.
43. Belcourt DR, Okawara Y, Fryer JN, Bennett HP: Immunocytochemical localization of granulin-I to mononuclear phagocytic cells of the teleost fish Cyprinus carpio and Carassius auratus. J Leukoc Biol 1995, 57( I):94-100.

44. Barreda DR, Hanington PC, Walsh CK, Wong P, Belosevic M: Differentially expressed genes that encode potential markers of goldfish macrophage development in vitro. Dev Comp Immunol 2004, 28(7-8):727-746.

45. Schultz RM: Regulation of zygotic gene activation in the mouse. BioEssays 1993, 15: 53|-538.

46. Daniel R, Daniels E, He Z, Bateman A: Progranulin (acrogranin/ PC cell-derived growth factor/granulin-epithelin precursor) is expressed in the placenta, epidermis, microvasculature, and brain during murine development. Dev Dyn 2003, 227(4):593-599.

47. Liau LM, Lallone RL, Seitz RS, Buznikov A, Gregg JP, Kornblum HI, Nelson SF, Bronstein JM: Identification of a human glioma-associated growth factor gene, granulin, using differential immuno-absorption. Cancer Res 2000, 60(5): $1353-1360$

48. Markert JM, Fuller CM, Gillespie GY, Bubien JK, McLean LA, Hong RL, Lee K, Gullans SR, Mapstone TB, Benos DJ: Differential gene expression profiling in human brain tumors. Physiol Genomics 200I, 5(I):2I-33.

49. Suzuki M, Yoshida S, Nishihara M, Takahashi M: Identification of a sex steroid-inducible gene in the neonatal rat hypothalamus. Neurosci Lett 1998, 242(3): 127-I 30.

50. Suzuki M, Nishiahara M: Granulin precursor gene: a sex steroidinducible gene involved in sexual differentiation of the rat brain. Mol Genet Metab 2002, 75(I):3I-37.

5I. Maack G, Segner H: Life-stage-dependent sensitivity of zebrafish (Danio rerio) to estrogen exposure. Comp Biochem Physiol C Toxicol Pharmacol 2004, I39( I-3):47-55.

52. Orkin SH, Zon LI: Genetics of erythropoiesis: induced mutations in mice and zebrafish. Annu Rev Genet 1997, 31:33-60.

53. Galloway JL, Zon LI: Ontogeny of hematopoiesis: examining the emergence of hematopoietic cells in the vertebrate embryo. Curr Top Dev Biol 2003, 53:139-I58.

54. Medvinsky A, Dzierzak E: Definitive hematopoiesis is autonomously initiated by the AGM region. Cell 1996, 86(6):897-906.

55. Bennett CM, Kanki JP, Rhodes J, Liu TX, Paw BH, Kieran MW, Langenau DM, Delahaye-Brown A, Zon LI, Fleming MD: Myelopoiesis in the zebrafish, Danio rerio. Blood 200I, 98: 643-65I.

56. $\mathrm{He} \mathrm{Z}$, Ong CH, Halper J, Bateman A: Progranulin is a mediator of the wound response. Nat Med 2003, 9(2):225-229.

57. Zhu J, Nathan C, Jin W, Sim D, Ashcroft GS, Wahl SM, Lacomis L, Erdjument-Bromage $H$, Tempst $P$, Wright $C D$, Ding $A$ : Conversion of proepithelin to epithelins: roles of SLPI and elastase in host defense and wound repair. Cell 2002, I I I(6):867-878.

58. Agabian N: Trans splicing of nuclear pre-mRNAs. Cell 1990, 6I: $1157-1160$.

59. Li BL, Li XL, Duan Z], Lee O, Lin S, Ma ZM, Chang CC, Yang XY, Park JP, Mohandas TK: Human acyl-CoA:cholesterol acyltransferase-I (ACAT-I) gene organization and evidence that the 4.3-kilobase ACAT-I mRNA is produced from two different chromosomes. J Biol Chem 1999, 274: I I060-I I07I.

60. Finta C, Zaphiropoulos PG: The human cytochrome P450 3A locus. Gene evolution by capture of downstream exons. Gene 2000, 260(I-2): 13-23.

61. Akopian AN, Okuse K, Souslova V, England S, Ogata N, Wood JN: Trans-splicing of a voltage-gated sodium channel is regulated by nerve growth factor. FEBS Lett 1999, 445(I): I77-182.

62. Bruzik JP, Maniatis T: Enhancer-dependent interaction between 5' and '3' splice sites in trans. Proc Natl Acad Sci U S A 1995, 92(15):7056-7059.

63. The Pfam database of protein families and HMMs [http:// pfam.wustl.edu/index.html]

64. European Bioinformatics Institute -Interpro [http:// www.ebi.ac.uk/interpro/]

65. Weijer CJ: Dictyostelium morphogenesis. Curr Opin Genet Dev 2004, I 4:392-398

66. Funk V, Kositsup B, Zhao C, Beers EP: The Arabidopsis xylem peptidase $X C P I$ is a tracheary element vacuolar protein that may be a papain ortholog. Plant Physiol 2002, I 28(I):84-94.

67. Wang $Y, G u X$ : Evolutionary patterns of gene families generated in the early stage of vertebrates. J Mol Evol 2000, $5 I(I): 88-96$. 
68. Bennett HPJ, Browne CA, Solomon S: Purification of the two major forms of rat pituitary corticotropin using only reversed-phase liquid chromatography. Biochemistry 1981, 20:4530-4538

69. Mullins MC, Hammerschmidt M, Hafter P, Nusslein-Volhard C: Large-scale mutagenesis in the zebrafish: in search of genes controlling development in a vertebrate. Curr Biol 1994, 4:189-202.

70. Kimmel CB, Ballard WW, Kimmel SR, Ullmann B, Schilling TF: Stages of embryonic development of the zebrafish. Dev Dyn 1995 , 203:253-310.

7I. Amemiya CT, Zon LI: Generation of a zebrafish PI artificial chromosome library. Genomics 1999, 58(2):21 I-213.

72. ZFIN [http://zfin.org/ZFIN/]

73. LocusLink [http://www.ncbi.nlm.nih.gov/LocusLink/]

74. Sambrook J, Fritsch EF, Maniatis T: Molecular cloning - A laboratory manual. Second edition. Cold Spring Harbor Laboratory Press; 1989.

75. Thisse C, Thisse B, Schilling TF, Postlethwait JH: Structure of the zebrafish snaill gene and its expression in wild-type, spadetail and no tail mutant embryos. Development 1993, I 19:1203-12I5.

76. DeBlock M, Debrouwer D: RNA-RNA in situ hybridisation using DIG-labeled probes: the effect of high molecular weight polyvinyl alcohol on the alkaline phosphatase indoxyl-nitoblue tetrazolium reaction. Anal Biochem 1993, 21 5:86-89.

77. Hedges SB: The origin and evolution of model organisms. Nat Rev Genet 2002, 3(II):838-849.

78. Pera EM, Hou S, Strate I, Wessely O, De Robertis EM: Exploration of the extracellular space by a large-scale secretion screen in the early Xenopus embryo. Int J Dev Biol 2005, 49(7):78I-796.

79. Bisbee CA, Baker MA, Wilson AC, Haji-Azimi I, Fischberg M: Albumin phylogeny for clawed frogs (Xenopus). Science 1977, 195(4280): 785-787.

Publish with Biomed Central and every scientist can read your work free of charge

"BioMed Central will be the most significant development for disseminating the results of biomedical research in our lifetime. "

Sir Paul Nurse, Cancer Research UK

Your research papers will be:

- available free of charge to the entire biomedical community

- peer reviewed and published immediately upon acceptance

- cited in PubMed and archived on PubMed Central

- yours - you keep the copyright

Submit your manuscript here:

http://www.biomedcentral.com/info/publishing_adv.asp
BioMedcentral 